\title{
Spectroscopic probes of isolated nonequilibrium quantum matter: Quantum quenches, Floquet states, and distribution functions
}

\author{
Yunxiang Liao* and Matthew S. Foster \\ Department of Physics and Astronomy, Rice University, Houston, Texas 77005, USA
}

(Received 27 June 2015; published 19 November 2015)

\begin{abstract}
We investigate radio-frequency (rf) spectroscopy, metal-to-superconductor tunneling, and angle-resolved photoemission spectroscopy (ARPES) as probes of isolated out-of-equilibrium quantum systems, and examine the crucial role played by the nonequilibrium distribution function. As an example, we focus on the induced topological time-periodic (Floquet) phase in a two-dimensional $p+i p$ superfluid, following an instantaneous quench of the coupling strength. The post-quench Cooper pairs occupy a linear combination of "ground" and "excited" Floquet states, with coefficients determined by the distribution function. While the Floquet band structure exhibits a single avoided crossing relative to the equilibrium case, the distribution function shows a population inversion of the Floquet bands at low energies. For a realization in ultracold atoms, these two features compensate, producing a bulk average rf signal that is well captured by a quasiequilibrium approximation. In particular, the rf spectrum shows a robust gap. The single crossing occurs because the quench-induced Floquet phase belongs to a particular class of soliton dynamics for the BCS equation. The population inversion is a consequence of this, and ensures the conservation of the pseudospin winding number. As a comparison, we compute the rf signal when only the lower Floquet band is occupied; in this case, the gap disappears for strong quenches. The tunneling signal in a solid-state realization is ignorant of the distribution function, and can show wildly different behaviors. We also examine rf, tunneling, and ARPES for weak quenches, such that the resulting topological steady state is characterized by a constant nonequilibrium order parameter. In a system with a boundary, tunneling reveals the Majorana edge states. However, the local $\mathrm{rf}$ signal due to the edge states is suppressed by a factor of the inverse system size, and is spatially deconfined throughout the bulk of the sample.
\end{abstract}

DOI: 10.1103/PhysRevA.92.053620

PACS number(s): 67.85.Lm, 03.75.Ss, 67.85.Hj

\section{INTRODUCTION}

\section{A. Idea and overview of results}

In recent years, there have been numerous studies on Floquet systems as they possess interesting topological phases absent in static systems [1-8]. Most of these are induced by external driving. For example, a Floquet topological insulator may arise when graphene is irradiated by circularly polarized light $[1,3,4,6,7]$. However, a topological Floquet phase can also be created in an isolated system such as a BCS superfluid following an instantaneous quench of the interaction strength [8-10]. The periodic modulation of the order parameter is self-generated by dynamics, and arises due to the single unstable mode associated to the BCS instability of the normal state [11-14].

In either of these two cases, the Floquet (quasienergy) band structure and the distribution function (nonequilibrium occupation number) are equally important. Much of the previous work focused on the connection between the band structure and topological properties [2,5], but did not take into account the distribution function which might be essential to determine experimental observables. The problem of irradiated graphene has been considered mainly in the Landauer-Büttiker formalism, wherein the mode occupation is fixed by ideal leads $[3,4,6,7]$. Even in that simplified setting, however, the relationship between the topology and Floquet edge states to measured transport coefficients is complicated, and the latter are not generically quantized [4,6,7]. More recent works have considered Floquet systems that are isolated or

\footnotetext{
*yliao@rice.edu
}

weakly coupled to the environment [15-17]. If external driving is applied to a system initially in its ground state, the particles will populate several Floquet bands, leading to a nontrivial distribution function.

To better understand the connection between the distribution function and observables, in this work we study the radiofrequency (rf) spectrum [18] of an isolated topological Floquet system. We consider the topological $p+i p$ Floquet superfluid induced by an instantaneous quench of interaction strength in a two-dimensional (2D) system of spinless fermions [8,9]. The long-time asymptotic steady state occupies two Floquet bands related by particle-hole symmetry. We determine the distribution function as well as the explicit BCS dynamics of the steady state, and derive the expression for the $\mathrm{rf}$ spectrum. This out-of-equilibrium system could be realized in an ultracold gas of fermionic atoms interacting through a $p$-wave Feshbach resonance [8,9].

Assuming it can also be realized in solid, we discuss the possibility of probing this nonequilibrium system by normalmetal-to-superconductor tunneling [19], whose setup closely parallels that of rf. Finally, we consider momentum-resolved spectra, as could be measured using angle-resolved photoemission spectroscopy (ARPES) [20]. Recent THz pump-probe experiments [21,22] have shown that order-parameter ("Higgs mode") quench dynamics can be induced in low-temperature superconductors on ultrashort time scales. Our results apply in a transient window over which the time-periodic phase can be stabilized, before pair breaking or other processes destroy it $[8,14]$.

We show that the bulk rf and tunneling spectra are in general very different, and this is tied to the nonequilibrium distribution function. The rf and tunneling spectra are both 
one-particle observables, but there is a crucial distinction [23]. In an ultracold gas, $\mathrm{rf}$ radiation can induce internal transitions in the atoms between states that participate in pairing and states that do not. The rf signal depends upon the occupation of both the paired and unpaired states. By contrast, tunneling from an idealized metal tip gives a current that is sensitive to the distribution function in the superconductor only on energy scales of order $\mathcal{E}_{F}$, the Fermi energy in the tip. The tunneling conductance at small bias is completely determined by the retarded single-particle Green's function in the Floquet phase. The same quantity determines transport in Floquet scattering theory [3], and signals the absence or presence of edge states in a system with a boundary $[8,9,24]$; however, it does not encode the occupation of bulk or edge states.

In our quenched-induced topological superfluid, we show that there is exactly one avoided crossing in the Floquet band structure. At the same time, there is a population inversion in the occupation of these bands, so that the "upper" ("lower") quasienergy band is occupied at low (high) energies. These two features compensate in the bulk rf prediction, so that the average signal is well approximated by that of a quasiequilibrium $p+i p$ superfluid. In particular, the spectrum exhibits a robust gap. Deviations from a quasiequilibrium picture appear as satellite coherence peaks, which have been studied previously [18]. To compare, we also consider the rf signal that obtains by populating only the lower Floquet band. In this case, the gap disappears for sufficiently strong quenches that induce large variations in the order parameter (i.e., strong driving). The tunneling signal is ignorant of the distribution function, and also depends sensitively on the quench. For strong quenches, there is no gap in the bulk tunneling spectrum. A quench-induced population inversion in a single-band Floquet system was studied in [25].

Unlike toy models sometimes considered in the literature, the drive frequency in our quench-induced Floquet system is much smaller than the bandwidth. This implies that there is significant folding of the unperturbed spectrum across the quasienergy zone. Although this situation should be the norm rather than the exception experimentally [1], it is usually more complicated to understand than the opposite, high- frequency limit $[3,5]$. In particular, one typically expects small gaps to open every time the spectrum crosses the zone, and this can complicate predictions for experiment [4].

By contrast, the presence of a single avoided band crossing in our Floquet band structure is a consequence of the BCS dynamics that generate it. In particular, the crossing arises because the quench-induced Floquet phase belongs to a particular class of soliton dynamics for the integrable BCS equations [12,13]. The population inversion is then a topological consequence of this since the texture of the instantaneous Anderson pseudospin description of the BCS state is conserved by the dynamics $[8,9]$. In other isolated topological Floquet systems, a population inversion is also expected for an odd number of avoided crossings.

We also consider the rf, tunneling, and ARPES signals that result from weaker quenches in the $p+i p$ superfluid, such that the order parameter asymptotes to a nonequilibrium constant value. In addition to bulk spectra, we consider a semi-infinite geometry with an edge. We show that while tunneling reveals the Majorana edge states, local rf spectroscopy is ill suited to find them. This is because the signal is suppressed by a factor of the system size, and is not spatially localized to the edge of the cloud.

\section{B. Review: Quench-induced topological Floquet phase in a $p+i p$ superfluid}

In this section, we briefly review the quench-induced nonequilibrium state [8,9] whose rf spectrum is investigated. The system is governed by the BCS Hamiltonian

$$
H=\sum_{\mathbf{k}} \frac{k^{2}}{2} c_{\mathbf{k}}^{\dagger} c_{\mathbf{k}}-2 G \sum_{\mathbf{k}, \mathbf{q}}^{\prime} \mathbf{k} \cdot \mathbf{q} c_{\mathbf{k}}^{\dagger} c_{-\mathbf{k}}^{\dagger} c_{-\mathbf{q}} c_{\mathbf{q}},
$$

where $c_{\mathbf{k}}$ annihilates a fermion with momentum $\mathbf{k}=\left\{k^{x}, k^{y}\right\}$ and mass $m=1$. The prime in $\sum^{\prime}$ indicates that the summation runs over momenta with non-negative $y$ components, i.e., $k^{y} \geqslant$ $0 . G>0$ is the interaction strength.

This Hamiltonian [Eq. (1.1)] has a $p+i p$ ground state whose order parameter assumes the form

$$
\begin{aligned}
\Delta(\mathbf{k}) & \equiv-2 G \sum_{\mathbf{q}}^{\prime} \mathbf{k} \cdot \mathbf{q}\left\langle c_{-\mathbf{q}} c_{\mathbf{q}}\right\rangle \\
& =\Delta_{0}\left(k^{x}-i k^{y}\right)=\Delta_{0} k e^{-i \phi_{k}},
\end{aligned}
$$

where $\phi_{k}$ is the polar angle of $2 \mathrm{D}$ vector $\mathbf{k} . \Delta_{0}$ is the orderparameter amplitude. The quasiparticle energy of such a $p+$ ip paired state is

$$
E_{k}=\sqrt{\left(\frac{k^{2}}{2}-\mu\right)^{2}+k^{2} \Delta_{0}^{2}} .
$$

Its spectrum is fully gapped as long as the chemical potential $\mu \neq 0$. The critical point $\mu=0$ separates two distinct topological phases: the weak pairing BCS phase $(\mu>0)$ and the strong pairing BEC phase $(\mu<0)$. The BCS phase is topologically nontrivial, i.e., if the system in this phase possesses a boundary, the Majorana edge state would appear. The squared order parameter $\left(\Delta_{0}\right)^{2}$ and chemical potential $\mu$ carry units of density; the fixed particle density $n$ sets the natural scale. All dimensionful quantities for a quench can be expressed in units of $n$, with at most logarithmic dependence upon an ultraviolet energy cutoff $\Lambda$ [9].

The system is initially prepared in the $p+i p$ ground state of the pre-quench Hamiltonian with interaction strength $G_{i}$. Then, the interaction strength is suddenly quenched to a different value $G_{f}$. After the quench, the system evolves as a superposition of many-body eigenstates of the post-quench Hamiltonian and acquires a steady state as $t \rightarrow \infty$.

In general, the asymptotic long-time evolution of the orderparameter amplitude can be expressed as

$$
\Delta(t)=\Delta_{\infty}(t) e^{-2 i \mu_{\infty} t},
$$

where $\Delta_{\infty}(t)$ could be 0 (phase I), a nonzero constant (phase II), or an oscillating function with time period $T$ (phase III), depending on the strength and direction of the quench $[8,9,13,26]$. In phase II, the real constant $\mu_{\infty}$ plays the role of a nonequilibrium chemical potential and determines the topological properties of the quench-induced state. It is topologically nontrivial (trivial) when $\mu_{\infty}>0\left(\mu_{\infty}<0\right)$. The out-of-equilibrium phase diagram is shown in Fig. 1 where 


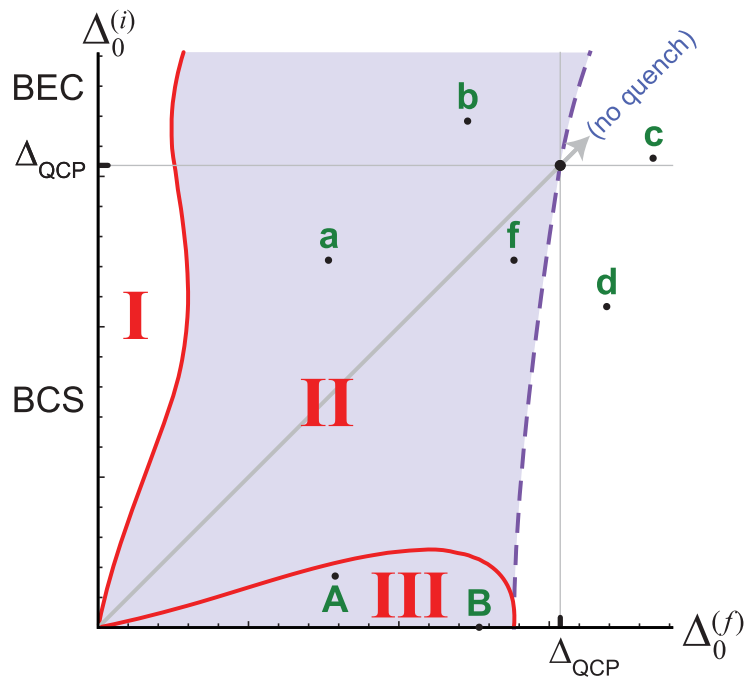

FIG. 1. (Color online) Quench phase diagram [31] showing three distinct dynamical phases characterizing the out-of-equilibrium states induced by sudden quench of the interaction strength in a $2 \mathrm{D}$ $p+i p$ fermion superfluid [8,9]. The order-parameter amplitude $\Delta_{\infty}(t)$ either vanishes, converges to a nonzero constant, or oscillates persistently for quenches in phases I, II, or III, respectively. The vertical and horizontal axes show $\Delta_{0}^{(i)}$ and $\Delta_{0}^{(f)}$, the order-parameter amplitudes associated to the ground states of the pre- and post-quench Hamiltonians. $\Delta_{\mathrm{QCP}}$ indicates the critical point where the ground-state chemical potential vanishes. It separates the topologically nontrivial $\mathrm{BCS}$ and trivial BEC phases in equilibrium. The purple dashed curve is its nonequilibrium extension. Each point to the left (right) of this line in II represents a topologically nontrivial (trivial) nonequilibrium state with positive (negative) $\mu_{\infty}$. Phase III is a quench-induced Floquet topological superfluid state, which is our main focus here. The labeled points are particular quenches discussed in the text. The amplitudes $\Delta_{0}^{(i, f)}$ and $\Delta_{\mathrm{QCP}}$ are measured in units of the inverse length $\sqrt{n}$, where $n$ is the fixed particle density [9].

we use $\Delta_{0}^{(i)}$ and $\Delta_{0}^{(f)}$, the order-parameter amplitudes of preand post-quench Hamiltonians' ground states, to indicate the coordinates of the quench $[8,9]$.

The main interest in this work is phase III, which can be understood as a quench-induced Floquet topological superfluid phase [8]. For both phases II and III, we use the term "topological" to denote the presence or absence of Majorana edge modes in a sample with a boundary, as encoded in the single-particle retarded Green's function [8,9,24]. This notion of topology relates to the spectrum of excitations that can be reached from the quench-induced nonequilibrium state by the application of a weak spectral probe, i.e., a frequency-dependent measurement. There is a different notion of topology that describes the many-body wave function itself, which is here always a BCS state. This is the pseudospin winding number that can be observed by an equal-time measurement, such as time of flight, and which cannot change following a quench. The conserved pseudospin winding does not determine whether or not edge states will appear; it instead encodes the occupation of the states [8,9]. Invariance of the winding number of the state was also noted in externally driven Floquet [17] and quenched topological [27] models.
In the following, we focus on weak-to-strong quenches within phase III where the order-parameter amplitude is given by a Jacobi elliptic function [9]. The constant $\Delta_{\infty}$ in phase II can be considered as a trivial case with vanishingly small oscillation amplitude.

\section{Floquet states and distribution function}

The order-parameter amplitude $\Delta_{\infty}(t)$ is determined by the self-consistent BCS dynamics [9,12,13,26,28,29]. The dynamics of individual Cooper pairs or (pairs of quasiparticle excitations) can then be obtained by solving the effective mean field Hamiltonian,

$$
\begin{aligned}
H_{\mathrm{BdG}}= & \sum_{\mathbf{k}}^{\prime}\left\{\left(\frac{k^{2}}{2}-\mu_{\infty}\right)\left(c_{\mathbf{k}}^{\dagger} c_{\mathbf{k}}+c_{-\mathbf{k}}^{\dagger} c_{-\mathbf{k}}\right)\right. \\
& \left.+k \Delta_{\infty}(t) c_{\mathbf{k}}^{\dagger} c_{-\mathbf{k}}^{\dagger}+k \Delta_{\infty}^{*}(t) c_{-\mathbf{k}} c_{\mathbf{k}}\right\} .
\end{aligned}
$$

Here, we have absorbed the polar phase of $\Delta(\mathbf{k})$ into the fermion operators via $c_{-\mathbf{k}} c_{\mathbf{k}} \rightarrow e^{-i \phi_{\mathbf{k}}-i 2 \mu_{\infty} t} c_{-\mathbf{k}} c_{\mathbf{k}}$. This transformation also boosts into the "rotating frame," so that $\mu_{\infty}$ is transferred from the order-parameter amplitude to the kinetic term.

The corresponding many-body wave function is given by the BCS product state

$$
|\Psi(t)\rangle=\prod_{\mathbf{k}}^{\prime}\left[u_{\mathbf{k}}(t)+v_{\mathbf{k}}(t) c_{\mathbf{k}}^{\dagger} c_{-\mathbf{k}}^{\dagger}\right]|0\rangle,
$$

where the time-dependent coherence factors follow the Bogoliubov-de Gennes (BdG) equation

$$
i \frac{d}{d t}\left[\begin{array}{c}
u_{\mathbf{k}}(t) \\
v_{\mathbf{k}}(t)
\end{array}\right]=\left[\begin{array}{cc}
-\frac{k^{2}}{2}+\mu_{\infty} & k \Delta_{\infty}^{*}(t) \\
k \Delta_{\infty}(t) & \frac{k^{2}}{2}-\mu_{\infty}
\end{array}\right]\left[\begin{array}{c}
u_{\mathbf{k}}(t) \\
v_{\mathbf{k}}(t)
\end{array}\right] .
$$

When $\Delta_{\infty}(t)$ is periodic in time as in phase III [8] with $\Delta_{\infty}(t+T)=\Delta_{\infty}(t)$, Eq. (1.7) has a pair of Floquet solutions. These are states taking the form $\left|\Psi^{(F)}(t)\right\rangle=\left|\Phi^{(F)}(t)\right\rangle e^{i E^{(F)} t}$, where $\left|\Phi^{(F)}(t)\right\rangle$ shares the same period $T$ with $\Delta_{\infty}(t)$, $\left|\Phi^{(F)}(t+T)\right\rangle=\left|\Phi^{(F)}(t)\right\rangle$. The quasienergy $E^{(F)}$ is defined up to integer multiples of the frequency $\Omega=2 \pi / T$. A Floquet state is a solution subject to the special initial condition that $\left|\Psi^{(F)}(0)\right\rangle$ is an eigenstate of time evolution operator $U(T)$ over one period: $U(T)\left|\Psi^{(F)}(0)\right\rangle=e^{i E^{(F)} T}\left|\Psi^{(F)}(0)\right\rangle$.

We will always assume a particular type of physical initial condition for the many-fermion system, which is the $p+i p$ ground state of the pre-quench Hamiltonian. The coherence factors in the asymptotic steady state are then given by a superposition of two Floquet states, both of which solve the BdG equation (1.7), that is,

$$
\begin{aligned}
{\left[\begin{array}{c}
u_{\mathbf{k}}(t) \\
v_{\mathbf{k}}(t)
\end{array}\right]=} & \sqrt{\frac{1-\gamma_{\mathbf{k}}}{2}}\left[\begin{array}{c}
u_{\mathbf{k}}^{(F)}(t) \\
v_{\mathbf{k}}^{(F)}(t)
\end{array}\right] e^{+i E_{\mathbf{k}}^{(F)} t} \\
& +\sqrt{\frac{1+\gamma_{\mathbf{k}}}{2}}\left[\begin{array}{c}
v_{\mathbf{k}}^{*(F)}(t) \\
-u_{\mathbf{k}}^{*(F)}(t)
\end{array}\right] e^{-i E_{\mathbf{k}}^{(F)} t+i \Gamma_{\mathbf{k}}} .
\end{aligned}
$$

These two Floquet states are related by particle-hole symmetry, and the relative coefficients are set by the "Cooper pair distribution function" $\gamma_{\mathbf{k}}\left(-1 \leqslant \gamma_{\mathbf{k}} \leqslant 1\right)$, which determines 
the nonequilibrium occupation number $f_{\mathbf{k}}^{(\mathrm{QP})}$ of the Floquet bands $[9,18]$. These are related via

$$
f_{\mathbf{k}}^{(\mathrm{QP})}=\frac{1}{2}\left(1+\gamma_{\mathbf{k}}\right)
$$

so that $\gamma_{\mathbf{k}}=-1(+1)$ corresponds to the absence (presence) of a pair of excited quasiparticles occupying states $\pm \mathbf{k}$. This is different from the occupation of the bare fermions $\left\langle\left(c_{\mathbf{k}}^{\dagger} c_{\mathbf{k}}+\right.\right.$ $\left.\left.c_{-\mathbf{k}}^{\dagger} c_{-\mathbf{k}}\right)\right\rangle$.

We will demonstrate in the following sections that the distribution function possesses topological information of the pre- and post-quench states. It can be extracted from the rf spectroscopy but not from the superconductor-normal-metal tunneling experiment.

\section{Outline}

This paper is organized as follows. In Sec. II, we derive general expressions for rf spectroscopy, tunneling amplitudes, and ARPES employed to probe the quench-induced out-ofequilibrium system. In Sec. III, as a warmup we compute the rf, tunneling, and ARPES spectra associated with several quenches in phase II with various topological properties. We examine the possibilities of extracting the topological character of both pre- and post-quench states by analyzing the rf spectrum. In Sec. IV, we turn to the analysis of the Floquet phase III. We study thoroughly two representative phase III quenches: one located in the vicinity of the phase II-III boundary, while the other lies deep in the phase III with weak initial interaction strength. The former (latter) is characterized by small harmonic (large anharmonic) amplitude modulations in $\Delta_{\infty}(t)$. We present and discuss the results for the rf, tunneling, and ARPES spectra, and we also discuss the connections between the Floquet band structure and the integrable BCS dynamics. Finally, in Sec. V we compute the local rf signal of a phase II post-quench system with a boundary, looking for signatures of Majorana edge states. We give our conclusion in Sec. VI.

Expressions for $\mathrm{rf}$ and tunneling harmonics relevant to probing the Floquet phase are relegated to Appendix A. The detailed derivations of the explicit Floquet state wave functions as well as the distribution function associated with phase III quenches are given in Appendix B.

\section{RF, TUNNELING SPECTROSCOPY, AND ARPES}

\section{A. Hamiltonian and current}

In a rf experiment, we assume that the system under study is realized in an ultracold fermion gas that possesses two relevant hyperfine states: $|1\rangle$ which participates in pairing, and $|2\rangle$ which does not.

The Hamiltonian for atoms in the nonpairing state $|2\rangle$ is given by

$$
H_{0}^{(d)}=\sum_{\mathbf{k}}\left(\frac{k^{2}}{2}+\mathcal{E}_{2,1}\right) d_{\mathbf{k}}^{\dagger} d_{\mathbf{k}},
$$

where $\mathcal{E}_{2,1}$ denotes the atomic transition energy between the states $|2\rangle$ and $|1\rangle$. This energy separation is typically much larger than any relevant to the many-body dynamics. $d_{\mathbf{k}}$ annihilates an atom in state $|2\rangle$ with momentum $\mathbf{k}$. In a $p$-wave paired superfluid, the $\mathrm{rf}$ radiation induces a transition in an atom with momentum $\mathbf{k}$ from state $|1\rangle$ to $|2\rangle$; an unpaired state |1) atom with momentum $-\mathbf{k}$ is left behind. This process is described by the transition (coupling) Hamiltonian

$$
H_{T}=\mathcal{T} \sum_{\mathbf{k}}\left[e^{i \omega_{L} t} c_{\mathbf{k}}^{\dagger} d_{\mathbf{k}}+e^{-i \omega_{L} t} d_{\mathbf{k}}^{\dagger} c_{\mathbf{k}}\right],
$$

where $\omega_{L}$ is the frequency of the rf field.

We will consider two observables: the local rf current $I\left(\mathbf{r}_{0}\right) \equiv\left\langle d n_{d}\left(\mathbf{r}_{0}\right) / d t\right\rangle$ and the global current $I \equiv\left\langle d N_{d} / d t\right\rangle$. Here, $n_{d}\left(\mathbf{r}_{0}\right)$ is the spatial density distribution of $|2\rangle$ atoms at position $\mathbf{r}_{0}$,

$$
n_{d}\left(\mathbf{r}_{0}\right) \equiv d^{\dagger}\left(\mathbf{r}_{0}\right) d\left(\mathbf{r}_{0}\right)=\sum_{\mathbf{k}_{1}, \mathbf{k}_{2}}\left[d_{\mathbf{k}_{1}}^{\dagger} d_{\mathbf{k}_{2}} e^{i\left(\mathbf{k}_{1}-\mathbf{k}_{2}\right) \mathbf{r}_{0}}\right],
$$

while $N_{d}$ is the total number of atoms in state $|2\rangle$

$$
N_{d}=\sum_{\mathbf{k}} d_{\mathbf{k}}^{\dagger} d_{\mathbf{k}} \text {. }
$$

Aside from the rf, we also consider normal-metal-tosuperconductor tunneling, assuming that the same post-quench asymptotic steady state can be hypothetically realized in a superconductor. The quenched condensate is brought into contact with a normal-metal tip, whose Hamiltonian is given by

$$
H_{0}^{(d)}=\sum_{\mathbf{k}}\left(\frac{k^{2}}{2}+V\right) d_{\mathbf{k}}^{\dagger} d_{\mathbf{k}} .
$$

Here, the tunneling bias $V$ is the energy of the tip relative to the condensate. We assume that the tip is ideal, characterized by a constant density of states $v_{0}$, and that prior to contact only states with momentum $k \leqslant q_{F}$ are occupied. The Hamiltonian

$$
\begin{aligned}
H_{T} & =\mathcal{T}\left[c^{\dagger}\left(\mathbf{r}_{0}\right) d\left(\mathbf{r}_{0}\right)+d^{\dagger}\left(\mathbf{r}_{0}\right) c\left(\mathbf{r}_{0}\right)\right] \\
& =\mathcal{T} \sum_{\mathbf{k}, \mathbf{q}}\left[c_{\mathbf{k}}^{\dagger} d_{\mathbf{q}} e^{i(\mathbf{k}-\mathbf{q}) \mathbf{r}_{0}}+d_{\mathbf{q}}^{\dagger} c_{\mathbf{k}} e^{-i(\mathbf{k}-\mathbf{q}) \mathbf{r}_{0}}\right]
\end{aligned}
$$

describes the tunneling between the metal tip and the superconductor at the contact point $\mathbf{r}_{0}$. The observable is again the local current at this point [Eq. (2.3)].

Comparing the Hamiltonian of tunneling [Eq. (2.6)] with that of the rf coupling [Eq. (2.2)] in the rotating frame $d_{\mathbf{k}} \rightarrow$ $d_{\mathbf{k}} e^{-i \omega_{L} t}$, the only difference is that while the contact is global for $\mathrm{rf}$, it is local for tunneling. They are identical if the transfer matrix element $\mathcal{T}$ in Eq. (2.6) is replaced with $\mathcal{T} \delta_{\mathbf{k}, \mathbf{q}}$, meaning the momentum is conserved in a rf experiment but not in tunneling.

\section{B. General $\mathrm{rf}$ and tunneling formulas}

Using linear-response theory, we obtain the rf current as a function of the coherence factors

$$
\begin{aligned}
I_{\mathrm{rf}}(t)= & 2 \mathcal{T}^{2} \operatorname{Re} \sum_{\mathbf{k}, \mathbf{q}} \delta_{\mathbf{k}, \mathbf{q}} \int_{-\infty}^{t} d t^{\prime} e^{i\left(\omega-\xi_{q}\right)\left(t^{\prime}-t\right)} \\
& \times\left[\left(1-f_{\mathbf{q}}^{(d)}\right) v_{\mathbf{k}}^{*}\left(t^{\prime}\right) v_{\mathbf{k}}(t)-f_{\mathbf{q}}^{(d)} u_{\mathbf{k}}^{*}(t) u_{\mathbf{k}}\left(t^{\prime}\right)\right],
\end{aligned}
$$

where $\omega \equiv \omega_{L}-\mathcal{E}_{2,1}$ is the detuning frequency, and $f_{\mathbf{k}}^{(d)}$ is the occupation number of atoms in state $|2\rangle$ before perturbed 
by $\mathrm{rf}$ radiation. The bare energy in the rotating frame is given by $\xi_{k}=k^{2} / 2-\mu_{\infty}$.

Similarly, we can obtain the expression for the tunneling current by replacing $\omega$ and $\delta_{\mathbf{k}, \mathbf{q}}$ in Eq. (2.7) with $-V$ and 1 , respectively. In addition, now $f_{\mathbf{q}}^{(d)}=\theta\left(q_{F}-q\right)$, where $\theta$ denotes the Heaviside unit step function. The differential conductance defined as $G(t) \equiv-\frac{d\left\langle I_{\tan }(t)\right\rangle}{d V}$ is given by

$$
\begin{aligned}
G(t)= & 2 \mathcal{T}^{2} v_{0} \operatorname{Re} \sum_{\mathbf{k}} \int_{-\infty}^{t} d t^{\prime} e^{i \tilde{V}\left(t^{\prime}-t\right)} \\
& \times\left[v_{\mathbf{k}}^{*}\left(t^{\prime}\right) v_{\mathbf{k}}(t)+u_{\mathbf{k}}^{*}(t) u_{\mathbf{k}}\left(t^{\prime}\right)\right] \\
= & -2 \mathcal{T}^{2} v_{0} \operatorname{Im} \sum_{\mathbf{k}} \int_{-\infty}^{t} d t^{\prime} e^{i \tilde{V}\left(t^{\prime}-t\right)} \mathcal{G}_{\mathbf{k}}^{(R)}\left(t, t^{\prime}\right),
\end{aligned}
$$

where $\tilde{V} \equiv-V-\xi_{q_{F}}$. On the last line, we have used the fact that

$$
\left[v_{\mathbf{k}}^{*}\left(t^{\prime}\right) v_{\mathbf{k}}(t)+u_{\mathbf{k}}^{*}(t) u_{\mathbf{k}}\left(t^{\prime}\right)\right]=\left\langle\left\{c_{\mathbf{k}}(t), c_{\mathbf{k}}^{\dagger}\left(t^{\prime}\right)\right\}\right\rangle=i \mathcal{G}_{\mathbf{k}}^{(R)}\left(t, t^{\prime}\right)
$$

is the single-particle retarded Green's function for a BCS product state.

The rf current [Eq. (2.7)] depends explicitly upon the distribution of the $|2\rangle$-state atoms and, as we will see, that of the paired ones as well. The tunneling conductance [Eq. (2.8)] by contrast depends only on the generalized spectral function (Wigner transform of the retarded Green's function) in the condensate.

\section{C. rf and tunneling for a Floquet system}

To find the rf signal from a topological Floquet system induced by phase III quench, we insert Eq. (1.8) into (2.7), and obtain the time-averaged rf current

$$
\begin{aligned}
\bar{I}_{\mathrm{rf}}= & \pi \mathcal{T}^{2} \sum_{n, \mathbf{k}}\left\{\left[\left(1-\gamma_{\mathbf{k}}\right)\left(1-f_{\mathbf{k}}^{(d)}\right)-\left(1+\gamma_{\mathbf{k}}\right) f_{\mathbf{k}}^{(d)}\right]\right. \\
& \times\left|\tilde{v}_{n, \mathbf{k}}\right|^{2} \delta\left(\omega-\xi_{\mathbf{k}}-E_{\mathbf{k}}^{(F)}+n \Omega\right) \\
& +\left[\left(1+\gamma_{\mathbf{k}}\right)\left(1-f_{\mathbf{k}}^{(d)}\right)-\left(1-\gamma_{\mathbf{k}}\right) f_{\mathbf{k}}^{(d)}\right] \\
& \left.\times\left|\tilde{u}_{n, \mathbf{k}}\right|^{2} \delta\left(\omega-\xi_{\mathbf{k}}+E_{\mathbf{k}}^{(F)}-n \Omega\right)\right\}
\end{aligned}
$$

where $\tilde{u}_{n, \mathbf{k}}$ and $\tilde{v}_{n, \mathbf{k}}$ are the Fourier coefficients of $u_{\mathbf{k}}^{(F)}(t)$ and $v_{\mathbf{k}}^{(F)}(t)$ :

$$
\left[\begin{array}{l}
u_{\mathbf{k}}^{(F)}(t) \\
v_{\mathbf{k}}^{(F)}(t)
\end{array}\right] \equiv \sum_{n=-\infty}^{\infty}\left[\begin{array}{c}
\tilde{u}_{n, \mathbf{k}} \\
\tilde{v}_{n, \mathbf{k}}
\end{array}\right] e^{-i n \Omega t}
$$

A version of Eq. (2.9) assuming $f_{\mathbf{k}}^{(d)}=0$ appeared previously in [18]. Here, all the oscillating terms are discarded since their time averages vanish. From an analogous calculation, we determine the time-averaged differential conductance

$$
\begin{array}{r}
\bar{G}=2 \pi \mathcal{T}^{2} \nu_{0} \sum_{n} \sum_{\mathbf{k}}\left[\left|\tilde{v}_{n, \mathbf{k}}\right|^{2} \delta\left(\tilde{V}-E_{\mathbf{k}}^{(F)}+n \Omega\right)\right. \\
\left.+\left|\tilde{u}_{n, \mathbf{k}}\right|^{2} \delta\left(\tilde{V}+E_{\mathbf{k}}^{(F)}-n \Omega\right)\right] .
\end{array}
$$

In a Floquet phase, the rf current and tunneling conductance also show periodic modulation at harmonics of the drive frequency $\Omega$. In Appendix A, we compute

$I_{\mathrm{rf}}(p) \equiv \int_{0}^{T} \frac{d t}{T} e^{i p \Omega t} I_{\mathrm{rf}}(t), G(p) \equiv \int_{0}^{T} \frac{d t}{T} e^{i p \Omega t} G(t)$.

These harmonic amplitudes provide additional observables that can be used to characterize a Floquet phase.

The time-averaged rf amplitude in Eq. (2.9) depends explicitly on the distribution function $\gamma_{\mathbf{k}}$, irrespective of the $|2\rangle$-state occupation $f_{\mathbf{k}}^{(d)}$. The same conclusion applies for all harmonics [Eq. (A1)]. By comparison, even the instantaneous tunneling conductance $G(t)$ is independent of $\gamma_{\mathbf{k}}$, if the tunneling probe has a constant density of states.

\section{ARPES formulas}

In ARPES experiments, one measures the Wigner transform of the single-particle lesser Green's function $\mathcal{G}_{\mathbf{k},<}\left(t, t^{\prime}\right)$ :

$$
S\left(\omega, \mathbf{k}, t_{0}\right) \equiv \int_{-\infty}^{\infty} d t e^{-i \omega t}(-i) \mathcal{G}_{\mathbf{k},<}\left(t_{0}-\frac{t}{2}, t_{0}+\frac{t}{2}\right),
$$

which equals

$$
-i \mathcal{G}_{\mathbf{k},<}\left(t, t^{\prime}\right) \equiv\left\langle c_{\mathbf{k}}^{\dagger}\left(t^{\prime}\right) c_{\mathbf{k}}(t)\right\rangle=v_{\mathbf{k}}^{*}\left(t^{\prime}\right) v_{\mathbf{k}}(t)
$$

Inserting Eq. (2.10) into (2.13) and (2.14), we arrive at the time-averaged ARPES signal from a quench-induced Floquet system

$$
\begin{aligned}
\bar{S}(\omega, \mathbf{k})= & \frac{\pi}{2} \sum_{n}\left[\left(1-\gamma_{\mathbf{k}}\right)\left|\tilde{v}_{n, \mathbf{k}}\right|^{2} \delta\left(-\omega-E_{\mathbf{k}}^{(F)}+n \Omega\right)\right. \\
& \left.+\left(1+\gamma_{\mathbf{k}}\right)\left|\tilde{u}_{n, \mathbf{k}}\right|^{2} \delta\left(-\omega+E_{\mathbf{k}}^{(F)}-n \Omega\right)\right] .
\end{aligned}
$$

\section{WEAK QUENCHES WITH CONSTANT ORDER PARAMETER: BULK SPECTROSCOPY IN PHASE II}

\section{A. Phase II introduction}

For the long-time asymptotic steady state following a phase II quench, only the zeroth-order Fourier coefficients $\tilde{u}_{0, \mathbf{k}}, \tilde{v}_{0, \mathbf{k}}$ are nonzero. The time-averaged rf current, tunneling conductance, and ARPES signal can be easily determined from Eqs. (2.9), (2.11), and (2.15), using the exact results for $\Delta_{\infty}$, $\mu_{\infty}$, and $\gamma(k)$ that can be obtained for a particular quench [9]. In what follows, unless otherwise stated, the nonpairing state is assumed to be initially unoccupied for all momenta in the $\mathrm{rf}$ calculation $f_{\mathbf{k}}^{(d)}=0$.

\section{B. Phase II: rf}

The rf spectrum of post-quench state with constant order parameter takes a nonzero value only within the region

$$
\begin{aligned}
& \omega \in\left[-2 \mu_{\infty},-\Delta_{\infty}^{2}\right] \cup[0, \infty), \mu_{\infty}>\frac{\Delta_{\infty}^{2}}{2} \\
& \omega \in\left[-\Delta_{\infty}^{2},-2 \mu_{\infty}\right] \cup[0, \infty), \frac{\Delta_{\infty}^{2}}{2}>\mu_{\infty}>0 \\
& \omega \in\left[-\Delta_{\infty}^{2}, 0\right] \cup\left[\left|2 \mu_{\infty}\right|, \infty\right), \mu_{\infty}<0 .
\end{aligned}
$$

It is composed of two continuous parts separated by a gap of width $\left|2 \mu_{\infty}\right|$ or $\Delta_{\infty}^{2}$. The gap appears for negative (positive) $\omega$ for topologically nontrivial BCS-type (trivial BEC-type) states with $\mu_{\infty}>0\left(\mu_{\infty}<0\right)[9,30]$. This feature in principle allows 
one to distinguish topological and trivial phases via a bulk measurement, even in the ground state [30].

A crucial difference from tunneling is that the weight always extends to zero on one side of the rf gap. This is due to the fact that fermions with $k \rightarrow 0$ are not coupled to the order parameter $\Delta$ for $p$-wave pairing, and thus transition between hyperfine states at the bare frequency.

The component on the positive (negative) detuning frequency side is due to the process where a $\mathrm{rf}$ photon with energy $\omega_{L}$ breaks the ground (excited) Cooper pair labeled by $k_{\omega}$ and excites one of the atoms to the different internal state $|2\rangle$, which does not participate in pairing. An unpaired state- $|1\rangle$ atom is left behind [9]. Here, $k_{\omega}=\sqrt{\frac{\omega\left(\omega+2 \mu_{\infty}\right)}{\omega+\Delta_{\infty}^{2}}}$ is the solution to $\omega= \pm E_{k}+\xi_{k}$, which comes from the conservation of energy. The term describing this process is weighted by $\frac{1-\gamma\left(k_{\omega}\right)}{2}\left[\frac{1+\gamma\left(k_{\omega}\right)}{2}\right]$, which reflects the occupation number of the ground (excited) Cooper pair.

As required by the conservation of pseudospin winding number $Q \equiv s_{z}(0)-s_{z}(\infty)$, the distribution function $\gamma(k)$ must wind from +1 at $k=0$ to -1 at $k \rightarrow \infty$ if the preand post-quench states are in different topological phases, i.e., if $\mu_{0}^{(i)} \mu_{\infty}<0$ [9]. When $\mu_{0}^{(i)} \mu_{\infty}>0, \gamma(k)$ does not wind but approaches -1 as $k \rightarrow\{0, \infty\}$. In particular, the difference between these two cases is the distribution function value at $k=0$ :

$$
\lim _{k \rightarrow 0} \gamma(k)= \begin{cases}-1, & \mu_{0}^{(i)} \mu_{\infty}>0, \\ +1, & \mu_{0}^{(i)} \mu_{\infty}<0 .\end{cases}
$$

The rf signal from which the distribution function can be extracted therefore possesses topological information of the state before and after the quench. A discontinuous jump at $\omega=0$ would appear in the rf spectrum if and only if $\mu_{0}^{(i)}>0$. This discontinuous jump exists at the right (left) side of gap edge if $\mu_{\infty}>0\left(\mu_{\infty}<0\right)$. On the other side of the gap, the spectrum grows continuously from 0 . This introduces the feasibility of using quantum quench in the detection of topological information of the system.

As an example, we evaluated the time-averaged rf spectra of the steady states induced by four different phase II quenches. These quenches are indicated as points "a" (BCS to BCS), "b" (BEC to BCS), "c" (BEC to BEC), and "d" (BCS to BEC) in Fig. 1. For quenches $\mathrm{a}$ and $\mathrm{b}$ (c and d), the asymptotic chemical potential $\mu_{\infty}>\Delta_{\infty}^{2} / 2\left(\mu_{\infty}<0\right)$, and the corresponding rf spectra are illustrated in Fig. 2 (Fig. 3). The gap spans from $-\Delta_{\infty}^{2}$ to 0 in Fig. 2, and from 0 to $-2 \mu_{\infty}$ in Fig. 3, as expected. In addition, the discontinuous jump appears at $\omega=0$ for quenches a and d, which obtain from a BCS initial state.

\section{Phase II: Tunneling}

As in rf, a gap appears in the tunneling spectrum. Different from rf, it spans the symmetric interval from $-E_{\min } \leqslant \tilde{V} \leqslant$ $E_{\min }$, where $E_{\min }$ is the minimum excitation energy (spectrum gap):

$$
\begin{gathered}
E_{\min }= \begin{cases}V_{\min }, & \mu_{\infty}>\Delta_{\infty}^{2}, \\
\mu_{\infty}, & \Delta_{\infty}^{2}>\mu_{\infty}>0, \\
-\mu_{\infty}, & \mu_{\infty}<0,\end{cases} \\
V_{\min } \equiv \Delta_{\infty} \sqrt{2 \mu_{\infty}-\Delta_{\infty}^{2}} .
\end{gathered}
$$
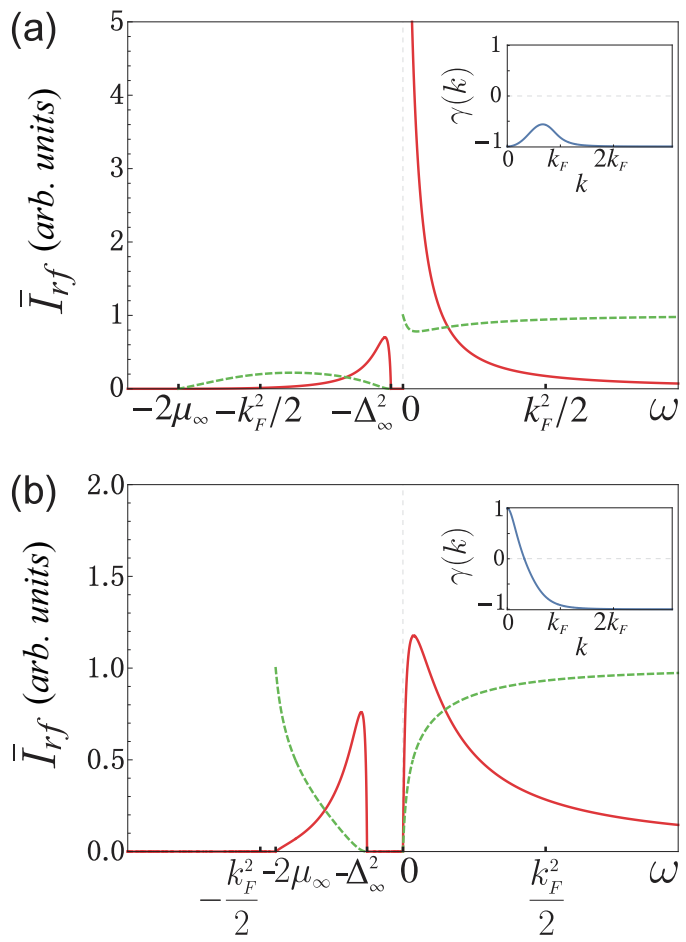

FIG. 2. (Color online) Bulk rf spectra (red solid curves) for nonequilibrium states induced by (a) BCS to BCS quench "a," (b) BEC to BCS quench "b," as indicated in the quench phase diagram Fig. 1. The green dashed curves show the relative weight imparted by the distribution function equal to $\frac{1-\gamma\left(k_{\omega}\right)}{2}, \frac{1+\gamma\left(k_{\omega}\right)}{2}$ in the positive- and negative-frequency domains, respectively. The distribution function $\gamma_{k}$ is illustrated in the inset. It winds from +1 at $k=0$ to -1 as $k \rightarrow \infty$ for the BEC to BCS quench $b$, but goes from -1 to -1 for BCS to BCS quench a. For both quenches, the gap is located between $-\Delta_{\infty}^{2}$ and 0 in the spectrum, indicating the (topologically nontrivial) weak-pairing BCS nature of the post-quench state. A discontinuous jump appears at $\omega=0$ in (a) which is associated to a quench from a BCS initial state. The coordinates for quenches a and $\mathrm{b}$ are $\left\{\Delta_{0}^{(i)}, \Delta_{0}^{(f)}\right\}=\{0.8,0.5\} \Delta_{\mathrm{QCP}}$ and $\{1.1,0.8\} \Delta_{\mathrm{QCP}}$, respectively.

Furthermore, the topological properties of the post-quench state can also be inferred from tunneling signal near the gap edge $\tilde{V}= \pm E_{\min }$. When $\mu_{\infty}>\Delta_{\infty}^{2}, \tilde{G}(\tilde{V})$ exhibits two coherence peaks around $\tilde{V}= \pm V_{\min }$, as shown in Fig. 4(a). The coherence peaks disappear for $\mu_{\infty}<\Delta_{\infty}^{2}$, and instead the spectrum shows a discontinuity on the positive (negative) edge of the gap for $\mu_{\infty}>0$ (BCS type) $\left[\mu_{\infty}<0\right.$ (BEC type)] [see Figs. 4(b) and 4(c)]. However, unlike the rf, the tunneling spectrum does not reveal information about the pre-quench state since the distribution function disappears from the differential conductance.

\section{Phase II: ARPES}

There are two branches in the ARPES spectrum of the post-quench state with constant order parameter. The upper (lower) one results from the excited (ground) Cooper pair, and is weighted by $\frac{1+\gamma\left(k_{\omega}\right)}{2}\left[\frac{1-\gamma\left(k_{\omega}\right)}{2}\right]$. For a ground state, the upper branch disappears. Similar to tunneling, there is no signal within the region $\omega \in\left(-E_{\min }, E_{\min }\right)$ [see Eq. (3.3)]. From Eq. (2.15), the intensity of the signal is determined by both 

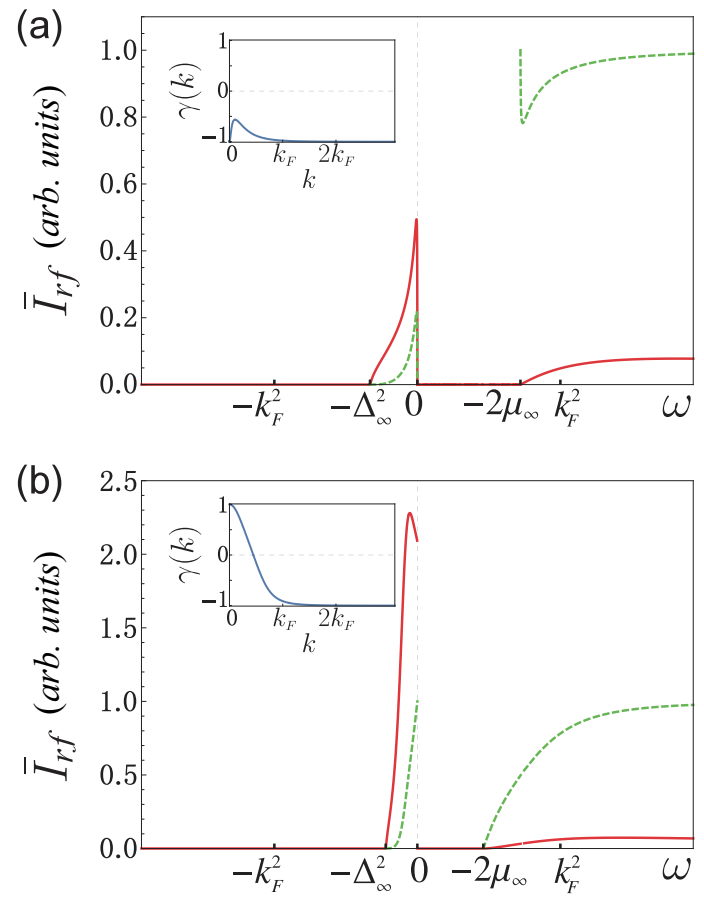

FIG. 3. (Color online) Bulk rf spectra for nonequilibrium states induced by (a) BEC to BEC quench "c," (b) BCS to BEC quench "d," as indicated in the quench phase diagram Fig. 1. As in Fig. 2, the red solid and green dashed curves respectively correspond to the actual spectrum and relative weight, while the inset shows the distribution function $\gamma_{k}$. The distribution function contains an even and odd number of zeros for quenches $\mathrm{c}$ and $\mathrm{d}$, respectively. The post-quench states corresponding to both (a) and (b) are topologically trivial, i.e., there would be no Majorana edge modes in a system with a boundary, and the nonequilibrium phase is of "BEC type." This is indicated by the gaps which appear between 0 to $\left|2 \mu_{\infty}\right|$. In (b), the rf spectrum exhibits a discontinuous jump at $\omega=0$, indicative of the BCS initial condition. The coordinates for quenches $\mathrm{c}$ and $\mathrm{d}$ are $\left\{\Delta_{0}^{(i)}, \Delta_{0}^{(f)}\right\}=\{1.02,1.2\} \Delta_{\mathrm{QCP}}$ and $\{0.7,1.1\} \Delta_{\mathrm{QCP}}$, respectively.

the distribution function $\gamma_{\mathbf{k}}$ and the moduli of the coherence factors $\left|\tilde{u}_{0, \mathbf{k}}\right|,\left|\tilde{v}_{0, \mathbf{k}}\right|$. Therefore, the distribution function is also measurable from an ARPES experiment.

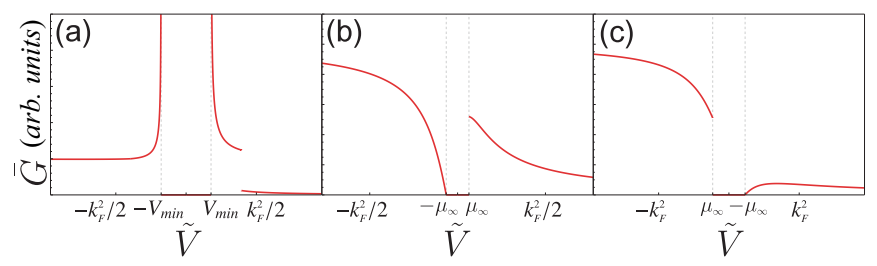

FIG. 4. (Color online) The bulk tunneling spectra of the nonequilibrium state following quenches (a) "a," (b) "f," (c) "d," as indicated in the quench phase diagram Fig. 1. The corresponding coordinates are $\left\{\Delta_{0}^{(i)}, \Delta_{0}^{(f)}\right\}=\{0.8,0.5\} \Delta_{\mathrm{QCP}},\{0.8,0.9\} \Delta_{\mathrm{QCP}}$, and $\{0.7,1.1\} \Delta_{\mathrm{QCP}}$, respectively. In (a), the long-time asymptotic nonequilibrium state has $\mu_{\infty}>\Delta_{\infty}^{2}$; two coherence peaks appear at $\tilde{V}= \pm V_{\min } . \Delta_{\infty}^{2}>$ $\mu_{\infty}>0$ and $\mu_{\infty}<0$ for quenches $\mathrm{f}$ and d, respectively. In (b), (c), the tunneling signal jumps from 0 at one edge of the gap $\tilde{V}=\mu_{\infty}$ and grows continuously at the other edge $\tilde{V}=-\mu_{\infty}$. The discontinuous jump occurs at the right (left) side of the gap in (b) [(c)].
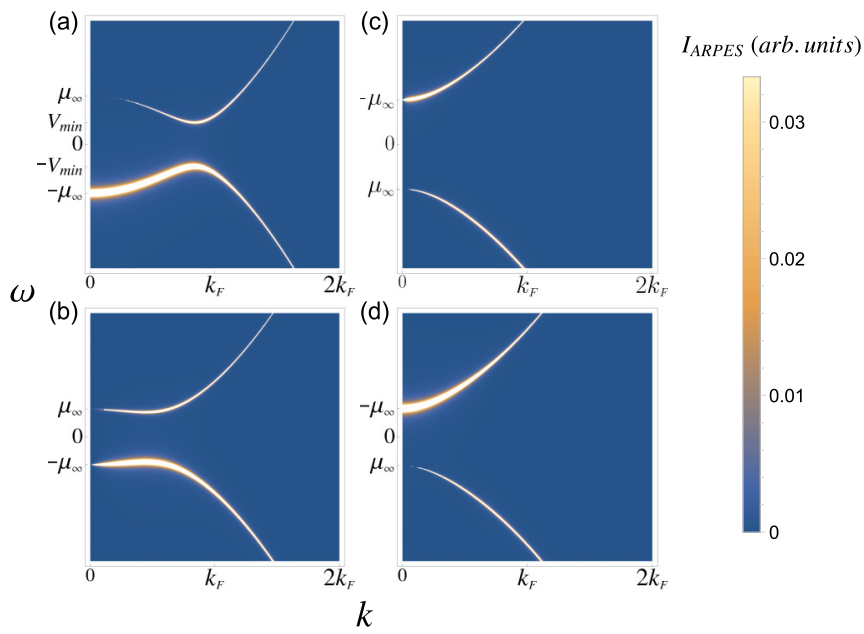

FIG. 5. (Color online) The ARPES spectra of the nonequilibrium state induced by quenches (a) "a," (b) "b," (c) “c," (d) "d."

In Fig. 5, we plot the ARPES signal for the steady state following phase II quenches $\mathrm{a}, \mathrm{b}$, c, and $\mathrm{d}$. To compute the signal, we replace the delta function in Eq. (2.15) by a smearing function $\delta_{s m}(x) \equiv \frac{\eta}{\pi\left(x^{2}+\eta^{2}\right)}$, and set $\eta=\left(6 \times 10^{-4}\right) \mathcal{E}_{F}$, where $\mathcal{E}_{F}=2 \pi n$ is the Fermi energy of the system ( $n$ is the density). For quenches a and $b$, the post-quench state is topologically nontrivial $\left(\mu_{\infty}>0\right)$ and, as a result, $\left|\tilde{u}_{0, \mathbf{k}}\right|=0$ at $k=0$. Examining the ARPES spectrum at $k=0$, we find it reaches maximum at $\omega=-\mu_{\infty}$ for BCS to BCS quench a [Fig. 5(a)] and disappears for BEC to BCS quench b [Fig. 5(b)]. The absence of signal for quench $\mathrm{b}$ at $k=0$ is due to the population inversion that occurs when the pre- and post-quench states are in different topological phases. On the other hand, the post-quench states induced by quenches $\mathrm{c}$ and $\mathrm{d}$ are topologically trivial $\left(\mu_{\infty}<0\right)$, and $\left|\tilde{v}_{0, \mathbf{k}}\right|=0$ at $k=0$. The ARPES signal with $k=0$ maximizes at $\omega=-\mu_{\infty}$ for BCS to BEC quench d [Fig. 5(d)] and vanishes for BEC to BEC quench c [Fig. 5(c)]. For quench d, the peak at $\omega=-\mu_{\infty}$ is a signature of the population inversion.

Based on the discussion above, we conclude that topological information of the state before and after the quench can be inferred from the ARPES signal. The pre-quench state is in BCS (BEC) phase when the signal is present (absent) at $k=0$. In addition, if the peak occurs at negative (positive) frequency, the post-quench state is topologically nontrivial (trivial).

\section{STRONG QUENCHES WITH PERIODIC ORDER PARAMETER: BULK SPECTROSCOPY IN FLOQUET PHASE III}

\section{A. Phase III introduction}

The problem of computing the long-time evolution of a paired fermion superfluid following a quantum quench can be reduced to solving the integrable dynamics of coupled Anderson pseudospins. Following a similar construction employed in the study of $s$-wave superfluids [12,13,26,28,29], in [9] a Lax spectral method is used to find the analytical solution for the 2D $p+i p$ model studied here. For a system of $N$ pseudospins, one introduces the spectral polynomial $Q_{2 N}(u)$, a conserved integral of motion for any value of $u$. In the limit $N \rightarrow \infty$, 
$Q_{2 N}(u)$, respectively, exhibits zero, one or two isolated pairs of roots in phases I, II, III of the quench phase diagram (Fig. 1). Isolated roots are those separated from the positive real axis in the complex $u$ plane. These always come in complex conjugate or negative real pairs. The remaining roots give rise to a branch cut along the positive real axis in the thermodynamic limit.

The isolated roots in turn parametrize the dynamics in an effective "reduced" problem of zero, one, or two collective pseudospins. The asymptotic evolution of the order parameter reaches a steady state described by Eq. (1.4), and this is completely determined by this reduced solution. The dynamics of individual pseudospins can then be reconstructed following a "bootstrap" procedure, solving the BdG equation (1.7) and exploiting the conservation of $Q_{2 N}(u)$ to determine the occupation of the nonequilibrium spectrum by ground- or excited-state pairs. The explicit solution for phases I and II was provided in [9]. The analogous calculation for phase III gives the combination of Floquet states in Eq. (1.8); the derivation is relegated to Appendix B.

For most quenches in phase III, the spectral polynomial possesses two complex conjugate pairs of isolated roots: one pair $u_{1, \pm} \equiv u_{1, \mathfrak{r}} \pm i u_{1, \mathfrak{i}}$ with $u_{1, \mathfrak{r}}>0$ exists only in this phase, while the other $u_{2, \pm} \equiv u_{2, \mathfrak{r}} \pm i u_{2, \mathfrak{i}}$ remains isolated in both phases II and III. Here, $u_{a, \mathfrak{r}}$ and $u_{a, \mathrm{i}}$ indicate the real and imaginary parts of $u_{a, \pm}, a=1,2$. We define

$$
\Delta_{a} \equiv \sqrt{\frac{\left|u_{a}\right|-u_{a, \mathfrak{r}}}{2}}, \quad \mu_{a} \equiv \frac{\left|u_{a}\right|}{2}, a=1,2
$$

with $\left|u_{a}\right| \equiv \sqrt{u_{a, \mathfrak{r}}^{2}+u_{a, \mathrm{i}}^{2}}$ the modulus of root $u_{a, \pm} . \Delta_{\infty}(t)=$ $\Delta_{\infty}(t+T)$ can be expressed in terms of the Jacobi elliptic function $\operatorname{cn}(z \mid M)$ and the four parameters $\Delta_{1,2}$ and $\mu_{1,2}$. The latter are functions of the phase III quench coordinates $\left\{\Delta_{0}^{(i)}, \Delta_{0}^{(f)}\right\}$, the fermion density $n$, and the ultraviolet cutoff energy $\Lambda$. The period $T$ is also determined by the isolated roots [8,9]. In terms of the quench $\left\{\Delta_{0}^{(i)}, \Delta_{0}^{(f)}\right\}$ for $\Delta_{0}^{(i)} \ll \Delta_{0}^{(f)}$ and $\Delta_{0}^{(f)} \lesssim \Delta_{\mathrm{QCP}}$, it is given by [8]

$$
T \sim \frac{2}{\Delta_{0}^{(f)} \sqrt{2 \mu_{0}^{(f)}-\left(\Delta_{0}^{(f)}\right)^{2}}} \ln \left[\frac{8 \pi n}{\Lambda} \frac{\Delta_{0}^{(f)} \sqrt{2 \mu_{0}^{(f)}-\left(\Delta_{0}^{(f)}\right)^{2}}}{\Delta_{0}^{(i)} \sqrt{2 \mu_{0}^{(i)}-\left(\Delta_{0}^{(i)}\right)^{2}}}\right],
$$

where $\mu_{0}^{(i)}$ and $\mu_{0}^{(f)}$ denote the ground-state chemical potentials corresponding to the pre- and post-quench Hamiltonians, respectively. In phase II, where $u_{2, \pm}$ is the only pair of isolated roots, $\mu_{\infty}=\mu_{2}$ and $\Delta_{\infty}=\Delta_{2}$, a constant.

In the complex $\Delta$ plane, the orbit of $\Delta_{\infty}(t)$ encircles $\Delta_{2}$ between the two turning points $\Delta_{2}+\Delta_{1}$ and $\Delta_{2}-\Delta_{1}$. Figure 6 shows the $\Delta_{\infty}(t)$ orbits of two different phase III quenches " $\mathrm{A}$ " and "B," as indicated in the quench phase diagram Fig. 1. The relatively weak quench $\mathrm{A}$ is close to phase II, and it exhibits a small orbit, with harmonic time dependence of the amplitude and phase. The other quench B is located deep in phase III. Since the oscillation amplitude is of the same order as the average value, this constitutes an example of "strong driving" with respect to the induced Floquet band structure. The amplitude and phase of the order parameter are strongly anharmonic in time for quench B. In the remainder of

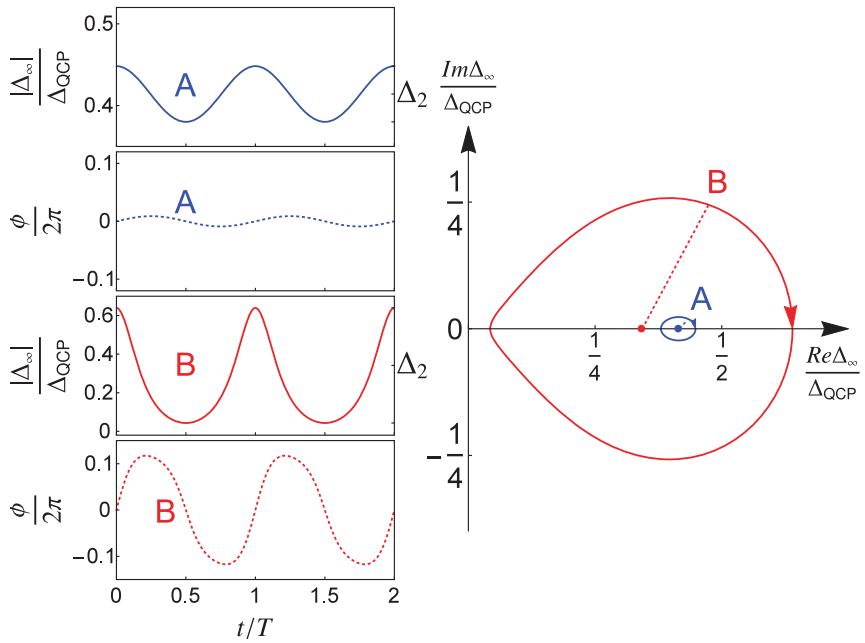

FIG. 6. (Color online) Oscillating order parameter $\Delta_{\infty}(t)$ induced by two phase III quenches, labeled " $\mathrm{A}$ " and "B" in the quench phase diagram Fig. 1. The left panels show the time dependence of the modulus and phase, while the right depicts the $\Delta_{\infty}(t)$ orbits in the complex $\Delta$ plane. The magnitude of $\left|\Delta_{\infty}(t)\right|$ oscillates between the two turning points $\Delta_{2} \pm \Delta_{1}$ [cf. Eq. (4.1)]. $\Delta_{2}$ is indicated by the center point of each orbit on the right. The coordinates for quenches $\mathrm{A}$ and $\mathrm{B}$ are $\left\{\Delta_{0}^{(i)}, \Delta_{0}^{(f)}\right\}=\{0.119,0.514\} \Delta_{\mathrm{QCP}}$, and $\{0.00651,0.825\} \Delta_{\mathrm{QCP}}$, respectively.

this section, we use these two points as representative quenches in phase III.

\section{B. Floquet band structure from BCS dynamics}

The explicit solution to the BCS dynamics of the Floquet system induced by a quench in phase III takes the form given by Eq. (1.8). It is a superposition of two Floquet states related by particle-hole symmetry. We determine the exact expressions for these Floquet states and the quasienergy spectrum in Appendix B. We also show that the modulus of the distribution function $|\gamma(k)|$ takes exactly the same form as previously obtained for phases I and II [9].

It will prove very useful to construct "phase II" (static quasiequilibrium) analogs of the Floquet states and quasienergies, which will serve as a reference point for comparison. We introduce

$$
\left[\begin{array}{l}
u_{2}(\varepsilon) \\
v_{2}(\varepsilon)
\end{array}\right] e^{i E_{2}(\varepsilon) t}, \quad\left[\begin{array}{c}
v_{2}(\varepsilon) \\
-u_{2}(\varepsilon)
\end{array}\right] e^{-i E_{2}(\varepsilon) t},
$$

which correspond to the ground and excited Cooper pair states for a phase II quench with $\Delta_{\infty}=\Delta_{2}$ and $\mu_{\infty}=\mu_{2}$. Here, we define

$$
\begin{aligned}
& E_{2}(\varepsilon) \equiv \sqrt{\left(\varepsilon / 2-\mu_{2}\right)^{2}+\varepsilon \Delta_{2}^{2}}, \\
& u_{2}(\varepsilon) \equiv \sqrt{\frac{1}{2}+\frac{\varepsilon / 2-\mu_{2}}{2 E_{2}(\varepsilon)}}, \\
& v_{2}(\varepsilon) \equiv-\sqrt{\frac{1}{2}-\frac{\varepsilon / 2-\mu_{2}}{2 E_{2}(\varepsilon)}} .
\end{aligned}
$$

In this section, we denote $\varepsilon \equiv k^{2}$ for modes with momentum $\pm \mathbf{k}$. 

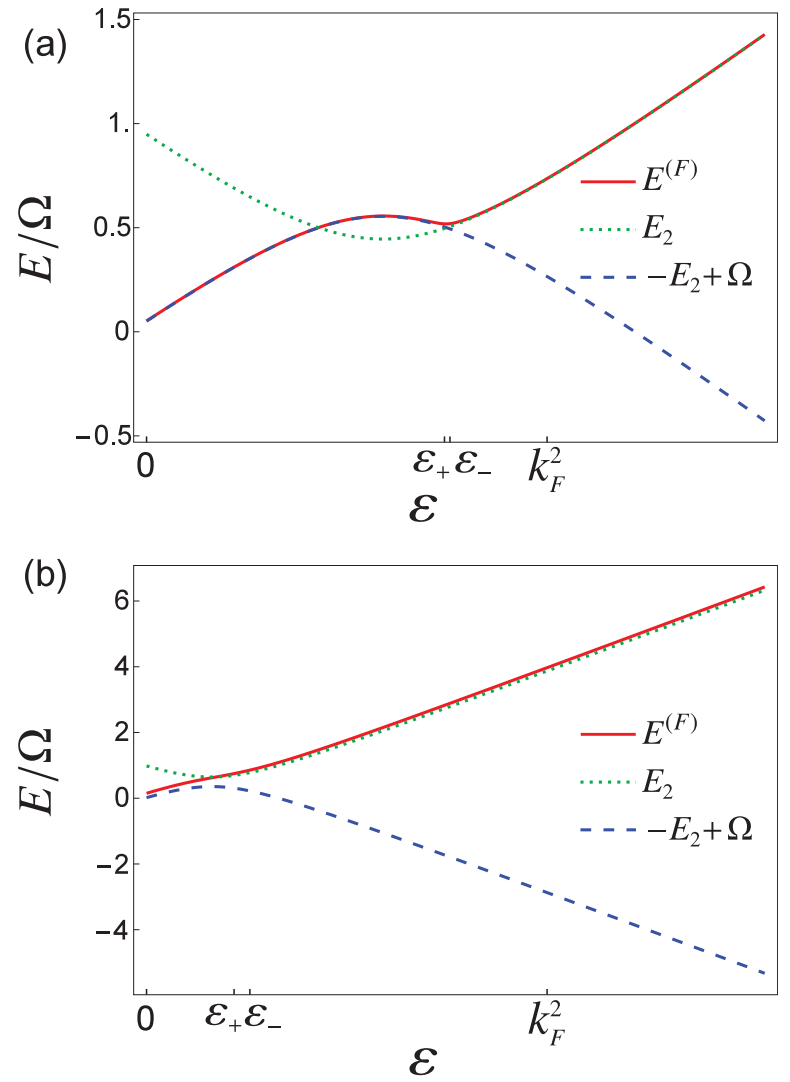

FIG. 7. (Color online) Quasienergy spectrum $E^{(F)}(\varepsilon)$ in the extended zone scheme for quenches (a) "A" and (b) "B", as indicated in the quench phase diagram Fig. 1 . Here, $\varepsilon=k^{2}$, where $k$ is the momentum. For both quenches, the quasienergy $E^{(F)}(\varepsilon)$ (red solid curve) is quite close to the static phase II approximation for the quasiparticle excitation energy $E_{2}(\varepsilon)$ (green dotted curve) for large $\varepsilon$, and to $-E_{2}(\varepsilon)+\Omega$ (blue dashed curve) for small $\varepsilon$. Here, $\Omega$ denotes the oscillation frequency of $\Delta_{\infty}(t)$, determined by the quench. The crossover of $E^{(F)}(\varepsilon)$ between "ground-state" and "excited-state" branches occurs around $\varepsilon_{ \pm}$, defined in the text.

In Fig. 7, we plot the quasienergy $E^{(F)}(\varepsilon)$ (red solid curve) as a function of $\varepsilon=k^{2}$ in the extended zone scheme for quenches $\mathrm{A}$ and $\mathrm{B}$. The green dotted curves in these figures show the dispersion of the excited states in the phase II approximation $E_{2}(\varepsilon)$, while the blue dashed curves are the groundstate energies shifted by the oscillation frequency $-E_{2}(\varepsilon)+\Omega$ [ $\Omega=2 \pi / T$, where $T$ is the quench-induced oscillation period of $\left.\Delta_{\infty}(t)\right]$. We find that in both cases the quasienergy spectrum exhibits a single "avoided crossing" or "Floquet band gap," in which the behavior of $E^{(F)}(\varepsilon)$ crosses over from the ground- to excited-state phase II approximations when the separation between $-E_{2}(\varepsilon)+\Omega$ and $E_{2}(\varepsilon)$ is small (Fig. 7). In particular, the quasienergy is close to $E_{2}(\varepsilon)$ when $\varepsilon \gg \varepsilon_{-}$, and to $-E_{2}(\varepsilon)+\Omega$ when $\varepsilon \ll \varepsilon_{+}$. Here, $\varepsilon_{ \pm}\left(\varepsilon_{+}<\varepsilon_{-}\right)$denote a pair of intermediate single-particle energies determined by the quench [see Eq. (B20) for explicit formulas]. Technically, $\varepsilon_{+}$ $\left(\varepsilon_{-}\right)$marks the energy at which the coherence factor $u^{(F)}(\varepsilon, t)$ $\left[v^{(F)}(\varepsilon, t)\right]$ reaches zero within one period $\left[u^{(F)}\left(\varepsilon_{+}, 0\right)=0\right.$, $v^{(F)}\left(\varepsilon_{-}, \frac{T}{2}\right)=0$; see Fig. 8, discussed following].

The coherence factors of the Floquet state $\left\{u^{(F)}(\varepsilon, t)\right.$, $\left.v^{(F)}(\varepsilon, t)\right\}$ oscillate little around those of the phase II
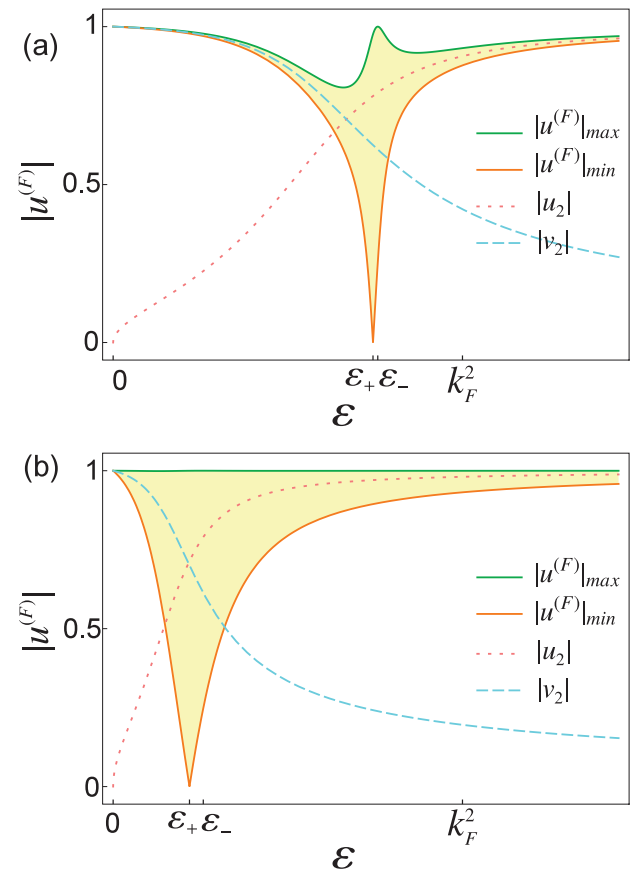

FIG. 8. (Color online) Oscillation of the first component of the phase III Floquet solution $\left|u^{(F)}(\varepsilon, t)\right|$, associated to quenches A (a) and B (b). The top green (bottom orange) solid curve shows the maximum (minimum) of $\left|u^{(F)}(\varepsilon, t)\right|$ within one period. The yellow (shaded) area enclosed by these two curves marks the region swept out by the periodic modulation. We compare these to the static phase II approximation for the coherence factors $\left|u_{2}(\varepsilon)\right|$ (pink dotted curve) and $\left|v_{2}(\varepsilon)\right|$ (cyan dashed curve). The magnitude of the Floquet component $\left|u^{(F)}(\varepsilon, t)\right|$ oscillates within a narrow region around the phase II approximation for most single-particle energies $\varepsilon$, and switches branches near $\varepsilon_{ \pm}$, where the oscillation amplitude is maximized.

approximation "ground state" $\left\{u_{2}(\varepsilon), v_{2}(\varepsilon)\right\}$ or "excited state" $\left\{v_{2}(\varepsilon),-u_{2}(\varepsilon)\right\} \mathrm{BdG}$ spinors for most single-particle levels, switching branches in the same region where the quasienergy changes behavior. Figure 8 depicts the oscillation of $\left|u^{(F)}(\varepsilon, t)\right|$ for quenches $\mathrm{A}$ and $\mathrm{B}$. The yellow (shaded) area marks the region swept out by $\left|u^{(F)}(\varepsilon, t)\right|$ in one period. It shows that $\left|u^{(F)}(\varepsilon, t)\right|$ undergoes small oscillations near $\left|v_{2}(\varepsilon)\right|$ (cyan dashed curve) or $\left|u_{2}(\varepsilon)\right|$ (pink dotted curve) when $\varepsilon \ll \varepsilon_{+}$or $\varepsilon \gg \varepsilon_{-}$, respectively.

As shown in Eq. (2.9) above, the Floquet band structure itself contains only part of the information relevant for $\mathrm{rf}$ spectroscopy. The other ingredient is the nonequilibrium distribution function $\gamma(\varepsilon)$, as appears in Eq. (1.8). This sets the weights of the "ground" and "excited" Floquet state solutions proportional to $\exp \left[ \pm i E^{(F)}(\varepsilon) t\right]$. The explicit formula for $\gamma(\varepsilon)$ is identical to phases I and II; the latter was obtained in [9]. In Appendix B, we confirm that the combination of Floquet and occupation factors in Eq. (1.8) gives the correct fixed particle density $n$ and self-consistent expression for $\Delta(t)$ in Eq. (1.4); see Eq. (B29).

We plot the distribution functions for quenches A and B in Fig. 9. Similar to the quasienergy spectrum and coherence factors, $\gamma(\varepsilon)$ exhibits a crossover from -1 at large $\varepsilon$ to +1 as $\varepsilon \rightarrow 0$. This holds true for any phase III quench, and implies that the nonequilibrium distribution function shows 

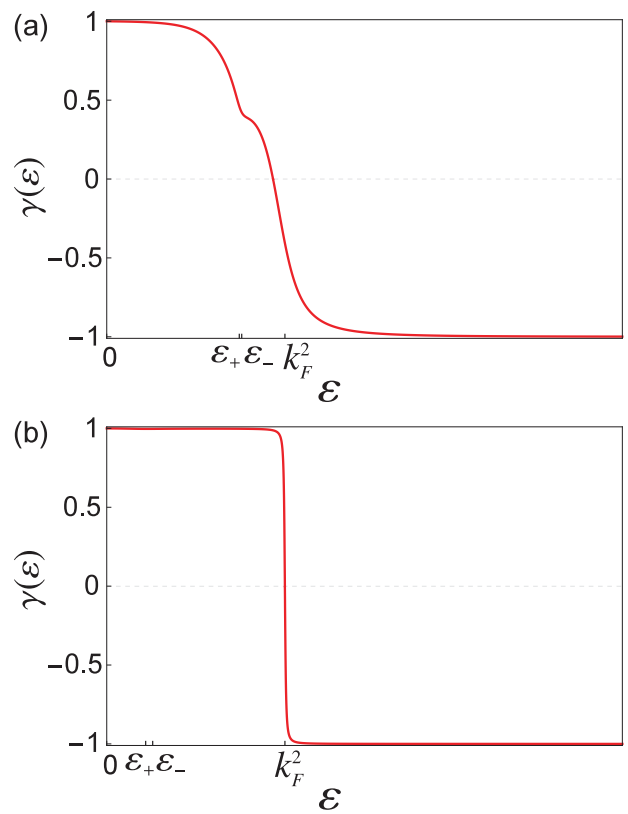

FIG. 9. (Color online) Distribution function $\gamma(\varepsilon)$ for quenches (a) A and (b) B. Due to the conservation of the pseudospin winding number [9], both wind from +1 at $\varepsilon=0$ to -1 at $\varepsilon \rightarrow \infty$.

a population inversion of the Floquet states at low energies, i.e., the "lower" Floquet band is occupied at large energies, but the "upper" one is filled for $k \lesssim k_{F}$ [cf. Eq. (1.9)].

In fact, the "winding" of $\gamma(\varepsilon)$ from -1 to +1 as $\varepsilon$ decreases from infinity is required by the topology. In particular, the pseudospin winding number that characterizes the instantaneous BCS state of the many-fermion system cannot change following a quench [9]. (This is different from the question of Majorana edge modes, which can appear or disappear; these are encoded in the retarded Green's function winding number $[8,9,24]$, and this quantity can change in a quench across the quantum critical point [9].) Given the single crossover of the quasienergy and Floquet coherence factors relative to the static quasiequilibrium case [phase II approximation, Eqs. (4.3) and (4.4)], conservation of the pseudospin winding number for BCS initial states implies that $\gamma(\varepsilon)$ must go to +1 as $\varepsilon \rightarrow 0$, so that $s_{\mathbf{k}}^{z}=\left\langle c_{\mathbf{k}}^{\dagger} c_{\mathbf{k}}+c_{-\mathbf{k}}^{\dagger} c_{-\mathbf{k}}-1\right\rangle / 2=+1 / 2$ at $\mathbf{k}=0$.

Taking into account both the quasienergy and coherence factors, we find that the Floquet state wave function in Eq. (1.8) $\left\{u^{(F)}(\varepsilon, t), v^{(F)}(\varepsilon, t)\right\} e^{i E^{(F)}(\varepsilon) t}$ can be well captured by the approximate phase II ground-state solution $\left\{u_{2}(\varepsilon), v_{2}(\varepsilon)\right\} e^{i E_{2}(\varepsilon) t}$ when $\varepsilon \gg \varepsilon_{-}$, and the excited state $\left\{v_{2}(\varepsilon),-u_{2}(\varepsilon)\right\} e^{-i E_{2}(\varepsilon) t}$ when $\varepsilon \ll \varepsilon_{+}$. This is true even for the strong quench $\mathrm{B}$. As a result, we can construct a full wave function for the phase II approximation as follows:

$$
\begin{aligned}
{\left[\begin{array}{l}
u^{(\mathrm{II})}(\varepsilon, t) \\
v^{(\mathrm{II})}(\varepsilon, t)
\end{array}\right] } & \equiv \sqrt{\frac{1-\gamma^{(\mathrm{II})}(\varepsilon)}{2}}\left[\begin{array}{c}
u_{2}(\varepsilon) \\
v_{2}(\varepsilon)
\end{array}\right] e^{i E_{2}(\varepsilon) t} \\
& +\sqrt{\frac{1+\gamma^{(\mathrm{II})}(\varepsilon)}{2}}\left[\begin{array}{c}
v_{2}(\varepsilon) \\
-u_{2}(\varepsilon)
\end{array}\right] e^{-i E_{2}(\varepsilon) t}, \\
\gamma^{(\mathrm{II})}(\varepsilon) & \equiv \operatorname{sgn}\left(\varepsilon-\varepsilon_{+}\right) \gamma(\varepsilon),
\end{aligned}
$$

where $\gamma(\varepsilon)$ is the true distribution function in phase III. The sgn function is necessary to "unwind" the distribution function since a phase II quench close to the II-III border has $\gamma(\varepsilon \rightarrow$ $0)=-1[9]$.

\section{Phase III dynamics, avoided crossing ("Floquet band gap"), and BCS instability of the normal state}

As discussed above, all phase III quenches feature a population imbalance in the basis of Floquet states at low momenta: the excited-state Floquet band becomes occupied with unit probability in the limit $\varepsilon=k^{2} \rightarrow 0$. This is depicted as the winding from -1 to +1 with decreasing $\varepsilon$ of the phase III distribution function $\gamma(\varepsilon)$ in Fig. 9. The mechanism for this is a combination of two factors. First, the Floquet band structure (quasienergy spectrum, and Floquet state coherence factors) exhibits exactly one "Floquet band gap" or avoided crossing, as shown in Figs. 7 and 8. In particular, the Floquet coherence factor $u^{(F)}(\varepsilon, t) \rightarrow 1\left[v^{(F)}(\varepsilon, t)\right] \rightarrow 0$ for both $\varepsilon \rightarrow$ $\{0, \infty\}$. By contrast, $u_{2}(\varepsilon)\left[v_{2}(\varepsilon)\right]$ approaches one (zero) for large $\varepsilon$, and zero (one) for $\varepsilon=0$; this is the usual behavior for coherence factors used to parametrize positive-energy quasiparticle excitations above a BCS ground state. In the Floquet case, the inversion $\left|u^{(F)}(\varepsilon, t)\right| \rightarrow v_{2}(\varepsilon)$ for $\varepsilon \ll \varepsilon_{+}$ implies the winding of $\gamma(\varepsilon)$, so as to ensure the conservation of the topological pseudospin winding number [9].

What is the origin of the single avoided crossing in the Floquet band structure? At first glance, this result is surprising, as we have obtained exact results in a system where the (quench-induced) drive frequency $\Omega$ is always much smaller than the bandwidth (which can be taken as an energy cutoff $\Lambda \gg k_{F}^{2} / 2$ [9]). The unperturbed (e.g., phase II approximate) spectrum folds many times when reduced to the first quasienergy Brillouin zone. We note that the periodic drive $\Delta_{\infty}(t)$ is not in general a pure harmonic (Fig. 6). We might therefore expect the opening of small band gaps whenever the folded spectrum approaches the zone edge, in analogy with $1 \mathrm{D}$ band structures in solid-state physics.

In fact, the single crossing can be understood as a consequence of the integrable BCS dynamics. The key idea is that any quench in phase III can be "adiabatically connected" to a special limiting case. This is the limit $\Delta_{0}^{(i)} \rightarrow 0^{+}$for fixed $\Delta_{0}^{(f)}$, which describes a quench from the Fermi liquid ground state perturbed by an infinitesimal seed of $p+i p$ [32] superfluid order. In this limit, the two isolated pairs of roots that characterize all phase III quenches coincide. The solution is a single soliton $[11,12,14]$ in the order parameter. For the $p+i p$ case, this takes the form

$$
\begin{aligned}
\Delta(t) & =\sqrt{\mathrm{R}(t)} \exp [-i \phi(t)], \\
\mathrm{R}(t) & =\frac{2 u_{\mathfrak{i}}^{2}}{|u| \cosh \left[2 u_{\mathfrak{i}}\left(t-t_{0}\right)\right]+u_{\mathfrak{r}}},
\end{aligned}
$$

where $u_{\mathfrak{r}, \mathfrak{i}}$ denote real and imaginary parts of the doubly degenerate isolated roots. This soliton solution describes the nonlinear collisionless dynamics of the Cooper pairs following a linear instability (exponential order-parameter growth) of the seeded Fermi liquid $[11,14] . \Delta(t)$ grows from the seed at $t \ll t_{0}$, reaching a maximum at $t=t_{0}$; it then decays again as $t \rightarrow \infty$. The peak magnitude is determined by the post-quench coupling strength; in terms of $\Delta_{0}^{(f)}$, one has

$$
u_{\mathfrak{r}} \simeq 4 \pi n, \quad u_{\mathfrak{i}} \simeq \sqrt{4 \pi n} \Delta_{0}^{(f)}, \quad \max \sqrt{\mathrm{R}(t)} \simeq \Delta_{0}^{(f)},
$$


valid for $\Delta_{0}^{(f)} \ll \Delta_{\text {QCP }}$. Here, $n$ denotes the fixed particle density. The decay $\Delta(t \rightarrow \infty)=0$ occurs because the quenched system cannot reach the preferred ground state (a paired BCS superfluid) without dissipating the large energy injected by the quench. Ultimately, pair breaking and other processes would induce thermalization, but we are neglecting these (see [8] for a discussion of the relevant time scales).

Mathematically, the soliton solution in Eq. (4.6) can be understood as arising due to the discontinuity in the initial spin distribution of the unperturbed Fermi step [12]. The only effect of nonzero $\Delta_{0}^{(i)}$ for a general phase III quench is to split the pairs of isolated roots, which gives rise to a train of solitons in time separated by a finite interval $T$ [see Eq. (4.2)]. By contrast, a normal-state initial distribution with multiple discontinuities is expected to seed a superposition of soliton trains with multiple incommensurate frequencies [12].

The explicit solution of the Floquet spinors given in Appendix B combined with Eq. (4.6) implies that

$$
\left|u^{(F)}(\varepsilon, t)\right| \rightarrow 1, \quad\left|v^{(F)}(\varepsilon, t)\right| \rightarrow 0,
$$

in the limit that $t \rightarrow \infty$, wherein the soliton has decayed. One can also verify that $|\gamma(\varepsilon)|=1$ for all $\varepsilon$ when $\Delta_{0}^{(i)} \rightarrow 0$. Thus, in the long-time limit we recover a pseudospin configuration consistent with a "normal state," $s^{z}(\varepsilon)= \pm 1 / 2$. This state must possess the same particle density as the initial Fermi step; moreover, given the connection between discontinuities and isolated roots discussed above, we expect only a single discontinuity in $s^{z}(\varepsilon)$. We conclude that the asymptotic pseudospin texture is that of the initial Fermi step. Different from a ground state, however, is the fact that this is encoded in the sign of the distribution function $\gamma(\varepsilon)$, rather than the coherence factors (since $\left|u^{(F)}(\varepsilon, t)\right|=1$ ). This is again because the quench-induced state is at all times very far from the preferred ground state. The relation $\left|u^{(F)}(\varepsilon, t)\right|=1$ for $\left|t-t_{0}\right| \rightarrow \pm \infty$ implies that the quasiparticle creation operator $\alpha_{\mathbf{k}}^{\dagger} \sim c_{\mathbf{k}}^{\dagger}$, where the latter creates an elementary fermion. In other words, all fermions are excitations for a quench that turns on pairing interactions, due to the BCS instability for any $k_{F}>0$. These characteristics of the nonequilibrium coherence factors and distribution function apply to all phase III quenches. As can be seen for the deep phase III quench "B" shown in Fig. 9(b), $\gamma(\varepsilon)$ approaches the Fermi step for small $\Delta_{0}^{i i}$.

\section{Phase III: rf spectroscopy}

In this section, we discuss the rf spectrum of the quenchinduced Floquet system and compare it with the phase II approximation [Eq. (4.5)]. Using the exact results for the Floquet coherence factors and the distribution function, we evaluate Eq. (2.9). The time-averaged rf currents (red solid curves) for quenches A and B are plotted in Fig. 10 together with the corresponding phase II approximations (blue dashed curves). As in Sec. III, here we assume that the nonpairing state is initially unoccupied for all momenta in the rf calculation, $f_{\mathbf{k}}^{(d)}=0$.

We expect to see two series of peaks evenly spaced by the quench-induced frequency of oscillation $\Omega$. These come from processes wherein a rf photon breaks a ground- or excited-state Cooper pair, and absorbs or emits several oscillation quanta [18]. No satellite peaks are visible for quench A, which is
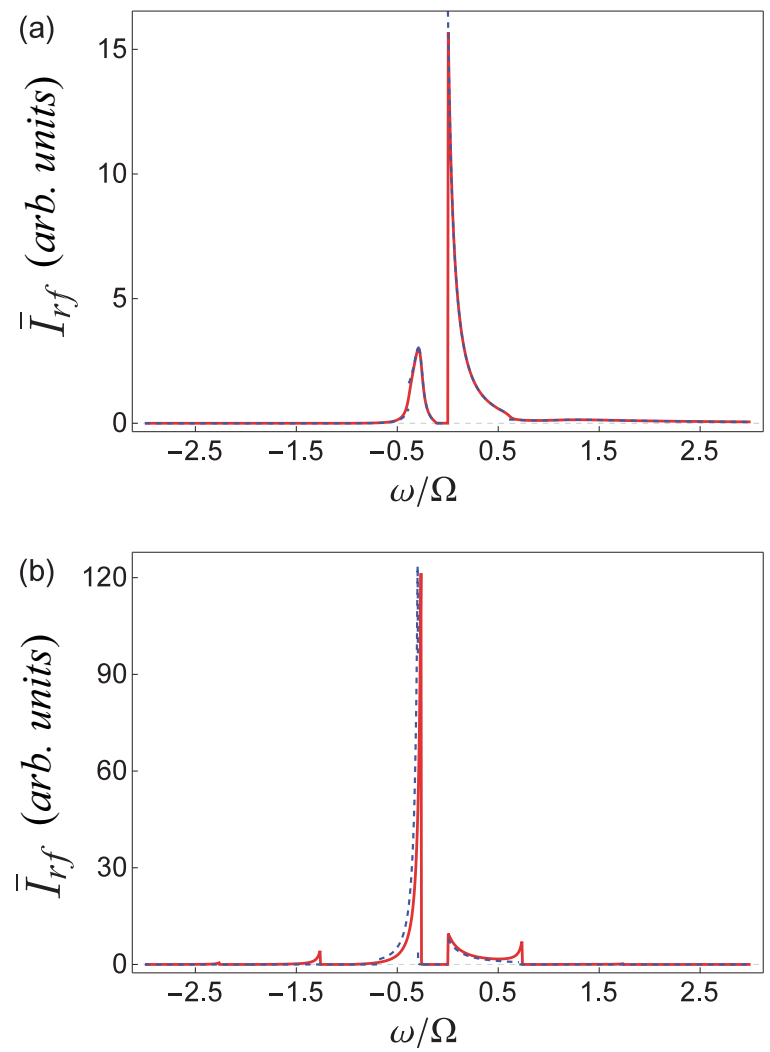

FIG. 10. (Color online) Time-averaged bulk rf spectra (red solid curves) from a topological Floquet system induced by the phase III quenches (a) "A" and (b) "B," as indicated in the quench phase diagram Fig. 1. For comparison, the corresponding phase II (static quasiequilibrium) approximations [Eq. (4.5)] are plotted with dashed blue curves. Unsurprisingly, the two curves agree well for quench A (a), which is close to the phase II-III border. More interesting is the close agreement for quench B (b), which resides deep in phase III and is characterized by the large anharmonic oscillations in $\Delta_{\infty}(t)$ shown in Fig. 6. The good agreement can be largely attributed to the nonequilibrium distribution functions. These show a population inversion in the Floquet bands at small $\varepsilon$, as shown in Fig. 9. The small deviation in (b) consists of a sequence of evenly spaced peaks (Floquet copies [18]), with the spacing equal to the oscillation frequency $\Omega$.

quite close to the phase II border and is characterized by a small oscillation amplitude (see Fig. 6). In this case, the signal is dominated by the zeroth-order term. The stronger quench $\mathrm{B}$ is deep in phase III; in this case, $\Delta_{\infty}(t)$ exhibits anharmonic time dependence and a large oscillation amplitude. Even for this quench, however, only the lowest-order satellite peaks are visible. This is because the oscillations in $u^{(F)}(\varepsilon, t)$ are confined to a particular region near $\varepsilon_{+} \lesssim \varepsilon \lesssim \varepsilon_{-}$, as shown in Fig. 8 .

In Fig. 10(a), the phase II approximation (blue dashed curve) agrees well with the actual rf spectrum for quench A (red solid curve). The deviation is also relatively small for quench B, depicted in Fig. 10(b). We note in particular that the bulk rf spectrum shows a robust gap, even for this strong phase III quench. We attribute this to the crucial role played by the distribution function. As discussed above, the topology of the initial pre-quench state constrains $\gamma(\varepsilon=0)=1$, so that the upper Floquet band is occupied at low energies. The inversion of $\gamma(\varepsilon)$ from -1 to +1 with decreasing $\varepsilon$ effectively 

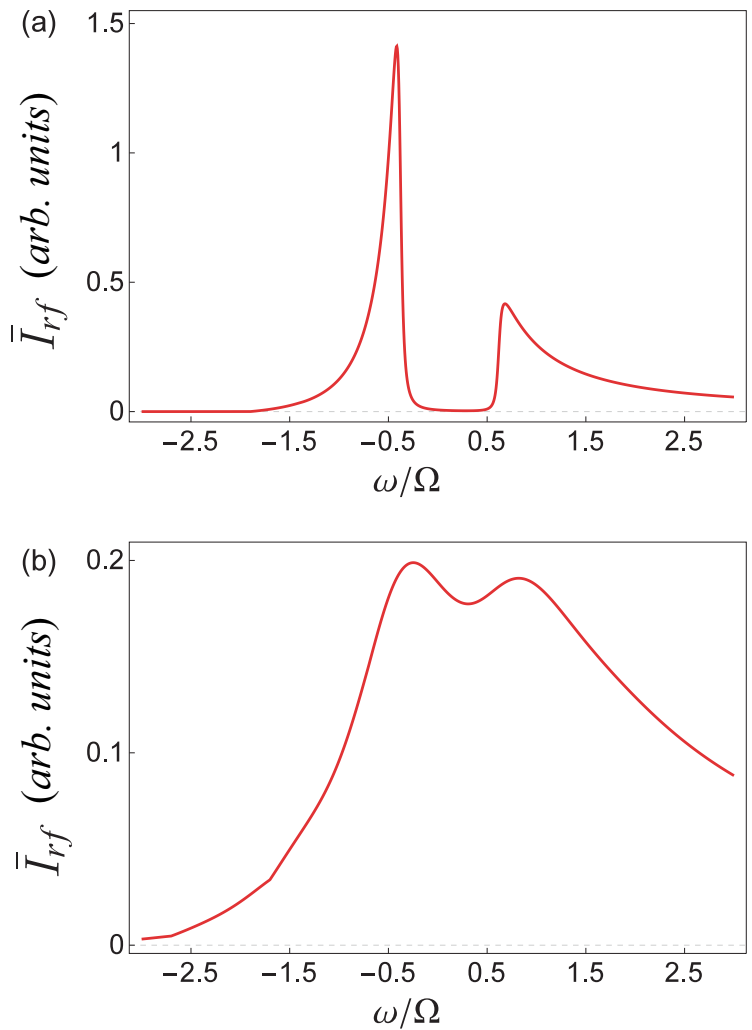

FIG. 11. (Color online) Time-averaged bulk rf spectra of the lower Floquet band associated to quenches (a) A and (b) B. These spectra are calculated using the same Floquet states and quasienergies utilized in Fig. 10 but assuming $\gamma(\varepsilon)=-1$, that is, only the lower Floquet band is occupied. Although these only differ in the distribution function, the spectra are completely unlike the corresponding ones in Fig. 10. In particular, the case (b) corresponding to the Floquet band structure induced by quench $\mathrm{B}$ gives a rf spectrum from the lower Floquet band that exhibits no gap, in contrast to quench-induced spectrum in Fig. 10(b), which takes into account the physical distribution function.

interchanges $u^{(F)}(\varepsilon, t)$ and $v^{(F)}(\varepsilon, t)$ in Eq. (1.8). Since $u^{(F)}(\varepsilon, t)$ behaves like $u_{2}(\varepsilon)\left[v_{2}(\varepsilon)\right]$ for $\varepsilon \gg \varepsilon_{ \pm}\left(\varepsilon \ll \varepsilon_{ \pm}\right)$, while $v^{(F)}(\varepsilon, t)$ shows the opposite behavior, the combination is well captured by Eq. (4.5).

To compare, we consider a Floquet system where the same oscillating order parameter is imposed externally. Unlike the quench-induced case, we assume that this system is prepared in a way so that only the lower Floquet band is occupied, i.e., $\gamma(\varepsilon)=-1$. The new time-averaged rf spectrum is shown in Fig. 11, and is found to be dramatically different from that of quench-induced asymptotic state. In particular, for the Floquet states and quasienergy spectrum associated to the strong quench $\mathrm{B}$, populating the lower Floquet band gives a bulk rf signal that does not exhibit a gap [Fig. 11(b)].

In addition to the time-averaged value, the rf signal in the Floquet phase exhibits harmonics at the drive frequency. In Fig. 12, we plot the real part of the first-order bulk rf harmonics signal $\operatorname{Re} I_{\mathrm{rf}}(1)$. This is defined via Eq. (2.12) and computed in Eq. (A1). In contrast to the average spectrum, a clear sequence of peaks is observed for both quenches.
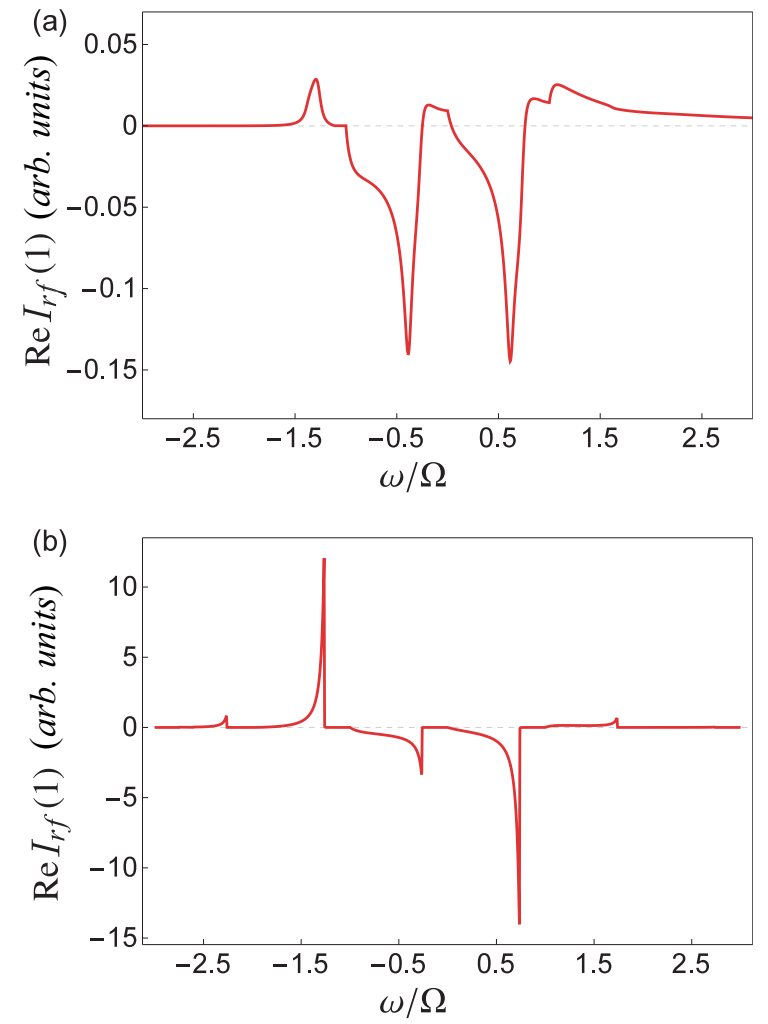

FIG. 12. (Color online) Real part of the bulk first-order rf harmonics $\operatorname{Re} I_{\mathrm{rf}}(1)$ for quenches (a) A, (b) B.

\section{E. Phase III: Tunneling}

Aside from the rf signal, we also compute the time-averaged tunneling conductance, assuming that phase III can be realized in a solid. For the quench A located in the vicinity of the phase II-III boundary, the phase II approximation works well for most $\tilde{V}$, as shown in Fig. 13(a). By contrast, it fails for quench B. In particular, the gap in the static quasiequilibrium phase II approximation does not exist in the real spectrum [see Fig. 13(b)]. These results should be compared to the bulk rf spectra, Figs. 10 and 11; the former uses the physical quench induced $\gamma(\varepsilon)$, while the latter assumes $\gamma(\varepsilon)=-1$. We conclude that the absence of a gap in the tunneling signal for quench B can be attributed to the fact that the latter is independent of $\gamma(\varepsilon)$ [Eq. (2.11)].

Figure 14 illustrates the harmonics of the tunneling conductance $\operatorname{Re} G(1)$ [Eq. (2.12)] for quenches A and B; these are computed via Eq. (A2). While clear peaks are observed for the weaker quench A similar to the rf shown in Fig. 12, the result is much less clear for the strong quench $\mathrm{B}$.

\section{F. Phase III: ARPES}

The time-averaged ARPES signals from the Floquet system induced by phase III quenches A and B are illustrated in Figs. 15(a) and 15(b), respectively. We find two series of Floquet copies in the spectra, and the intensity decreases fast as the Fourier order increases. For comparison, the phase II approximations associated with quenches A and B are respectively shown in Figs 15(c) and 15(d). We notice the phase II approximation resembles the leading-order copy 

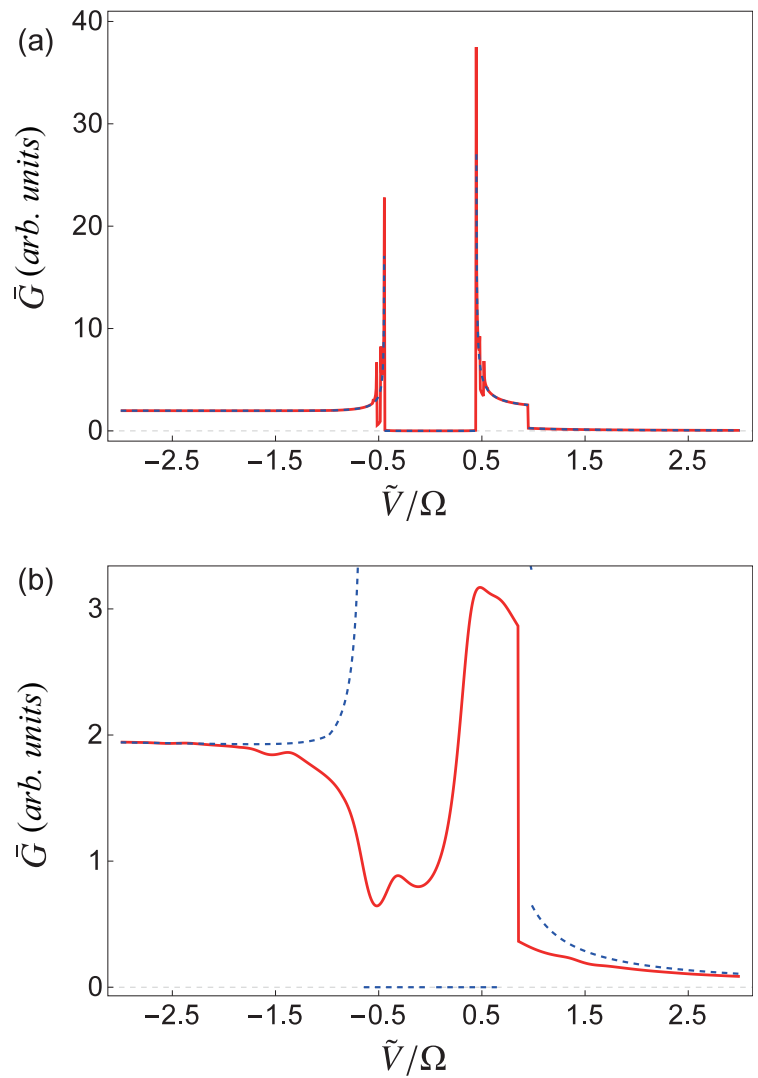

FIG. 13. (Color online) Time-averaged bulk tunneling conductance for a topological Floquet system following quenches (a) A, (b) B. The red solid curve and blue dashed curve in this figure respectively show the phase III conductance $\bar{G}(\tilde{V})$ and its phase II approximation. These two curves agree well in (a) but not in (b). The phase II approximation fails for the strong quench B as the distribution function does not influence the tunneling signal (cf. the rf spectra in Figs. 10 and 11).

in the ARPES signal for most momentum $k$, but not for those around $k_{+} \equiv \sqrt{\varepsilon_{+}}$[Eq. (B20)] where the wave function exhibits the biggest difference from its phase II approximation [Eq. (4.5)], especially for the relatively strong quench B.

\section{LOCAL BULK AND EDGE SPECTROSCOPY}

In this section, we look for experimental signatures of Majorana fermion edge modes $[33,34]$ in a $2 \mathrm{D} p+i p$ topological superfluid. Specifically, we investigate the contribution from the edge modes to the local rf and tunneling signals. Our results apply equally to the BCS ground state, and to a quench-induced topologically nontrivial superfluid in phase II. The latter is characterized by a constant $\Delta_{\infty}$ and $\mu_{\infty}>0$ [Eq. (1.4)]; quenches producing states of this type are indicated by the shaded portion of region II in Fig. 1.

The system is assumed to occupy an infinite strip of width $\mathcal{L}$ in the $x$ direction, extended in the $y$ direction. Our results also apply to the half-plane $\{(x, y) \mid x \geqslant 0, y \in \mathbb{R}\}$. At a physical edge $x=x_{\text {edge }}$, we impose hard wall boundary conditions.
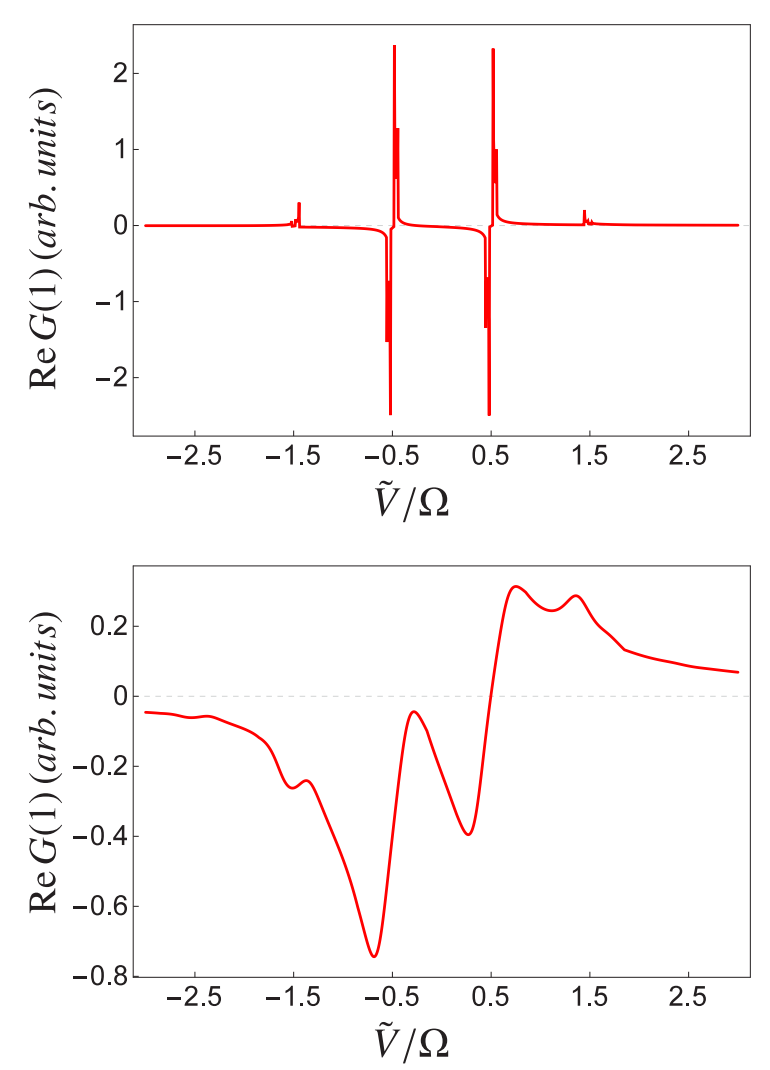

FIG. 14. (Color online) Real part of the bulk first-order tunneling harmonics $\operatorname{Re} G(1)$ for quenches (a) A, (b) B.

\section{A. BdG Hamiltonian}

The effective Bogoliubov-de Gennes mean field Hamiltonian in this geometry is given by

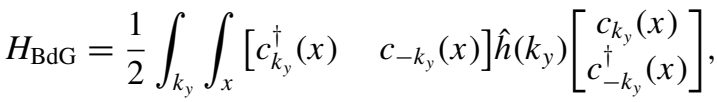

$$
\begin{aligned}
& \hat{h}\left(k_{y}\right)=\left[\begin{array}{cc}
-\frac{1}{2} \frac{d^{2}}{d x^{2}}+\frac{k_{y}^{2}}{2}-\mu_{\infty} & \Delta_{\infty}\left(-i \frac{d}{d x}-i k_{y}\right) \\
\Delta_{\infty}^{*}\left(-i \frac{d}{d x}+i k_{y}\right) & \frac{1}{2} \frac{d^{2}}{d x^{2}}-\frac{k_{y}^{2}}{2}+\mu_{\infty}
\end{array}\right] .
\end{aligned}
$$

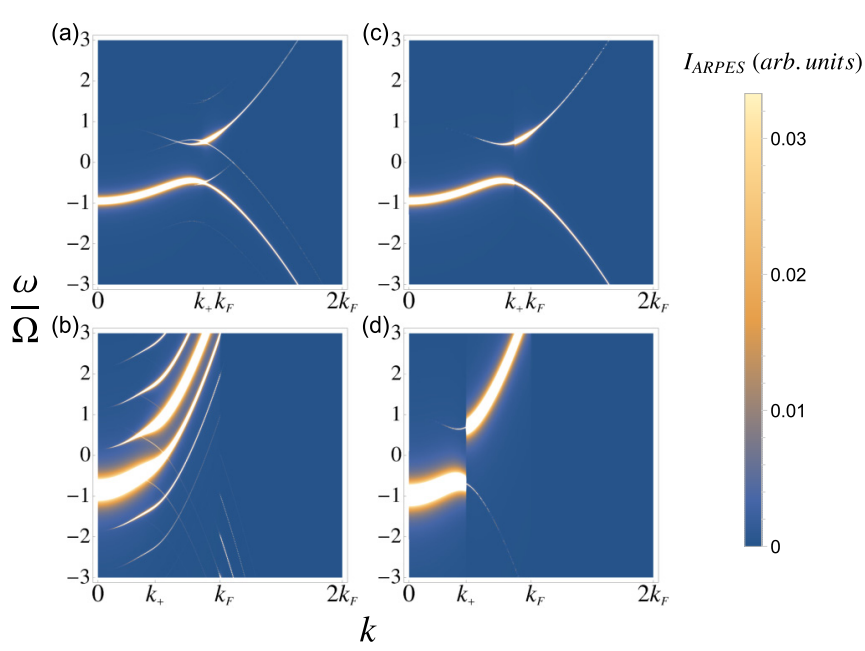

FIG. 15. (Color online) The ARPES spectra for the topological Floquet system induced by phase III quenches (a) A, (b) B. Their phase II approximations are depicted in (c) and (d), respectively. 
After the Bogoliubov transformation,

$$
\left[\begin{array}{c}
a_{k_{y}}(p) \\
a_{-k_{y}}^{\dagger}(p)
\end{array}\right]=\int_{x}\left[\begin{array}{cc}
u_{k_{y}}(p, x) & v_{k_{y}}(p, x) \\
v_{-k_{y}}^{*}(p, x) & u_{-k_{y}}^{*}(p, x)
\end{array}\right]\left[\begin{array}{c}
c_{k_{y}}(x) \\
c_{-k_{y}}^{\dagger}(x)
\end{array}\right],
$$

where the amplitudes satisfy

$$
\hat{h}^{*}\left(k_{y}\right)\left[\begin{array}{c}
u_{k_{y}}(p, x) \\
v_{k_{y}}(p, x)
\end{array}\right]=E_{k_{y}}(p)\left[\begin{array}{c}
u_{k_{y}}(p, x) \\
v_{k_{y}}(p, x)
\end{array}\right],
$$

with $E_{k_{y}}(p) \geqslant 0$, the Hamiltonian reduces to

$$
H_{\mathrm{BdG}}=\frac{1}{2} \int_{k_{y}} \sum_{p} E_{k_{y}}(p)\left[\begin{array}{c}
a_{k_{y}}^{\dagger}(p) a_{k_{y}}(p) \\
-a_{-k_{y}}(p) a_{-k_{y}}^{\dagger}(p)
\end{array}\right] .
$$

Here, $a_{k_{y}}(p)$ and $a_{k_{y}}^{\dagger}(p)$ are the annihilation and creation operators associated with the quasiparticle excitation labeled by $p$ and transverse momentum $k_{y}$. The corresponding quasiparticle energy is denoted as $E_{k_{y}}(p) . \sum_{p}$ runs over all the eigenstates with non-negative eigenvalues, including edge states (if present).

Atoms in the nonpairing hyperfine state $|2\rangle$ in the rf experiment are described by the Hamiltonian

$$
\begin{gathered}
H_{0}^{(d)}=\int_{k_{y}} \int_{x} d_{k_{y}}^{\dagger}(x) \hat{h}_{d}\left(k_{y}\right) d_{k_{y}}(x), \\
\hat{h}_{d}\left(k_{y}\right)=-\frac{1}{2} \frac{d^{2}}{d x^{2}}+\frac{k_{y}^{2}}{2}-\mu_{\infty}+\mathcal{E}_{2,1},
\end{gathered}
$$

which can be reduced to

$$
H_{0}^{(d)}=\int_{k_{y}} \sum_{k}\left[\xi_{k_{y}}(k)+\mathcal{E}_{2,1}\right] b_{k_{y}}^{\dagger}(k) b_{k_{y}}(k),
$$

where $b_{k_{y}}(k)$ annihilates an atom in state $|2\rangle$, labeled by $k$ and with energy $\xi_{k_{y}}(k)+\mathcal{E}_{2,1}$ :

$$
\begin{aligned}
& b_{k_{y}}(k)=\int_{x} w(k, x) d_{k_{y}}(x), \\
& \hat{h}_{d}^{*}\left(k_{y}\right) w(k, x)=\left[\xi_{k_{y}}(k)+\mathcal{E}_{2,1}\right] w(k, x) .
\end{aligned}
$$

Here, $\xi_{k_{y}}(k)=\left(k^{2}+k_{y}^{2}\right) / 2-\mu_{\infty}$. The wave function $w(k, x)$ for the nonpairing state is a real sinusoid that vanishes at the edges of the cloud. In what follows, for clarity we use $p$ and $k$ to indicate the excitation in the paired and unpaired atomic levels, respectively.

\section{B. Local rf current}

From the Heisenberg equation of motion (EOM), the local current at $\mathbf{r}_{0}$ equals

$$
\begin{aligned}
I\left(\mathbf{r}_{0}, t\right) & \equiv \frac{d n_{d}\left(\mathbf{r}_{0}, t\right)}{d t}=i\left[H, n_{d}\left(\mathbf{r}_{0}\right)\right], \\
H & =H_{\mathrm{BdG}}+H_{0}^{(d)}+H_{T} .
\end{aligned}
$$

The local current $I\left(\mathbf{r}_{0}, t\right)$ therefore is composed of two components from the commutator with $H_{T}$ and $H_{0}^{(d)}$, respectively:

$$
\begin{aligned}
I\left(\mathbf{r}_{0}\right) & =I_{T}\left(\mathbf{r}_{0}\right)+I_{d}\left(\mathbf{r}_{0}\right), \\
I_{T}\left(\mathbf{r}_{0}\right) & \equiv i\left[H_{T}, n_{d}\left(\mathbf{r}_{0}\right)\right], \\
I_{d}\left(\mathbf{r}_{0}\right) & \equiv i\left[H_{0}^{(d)}, n_{d}\left(\mathbf{r}_{0}\right)\right] .
\end{aligned}
$$

In the interaction picture, the current expectation value is given by

$$
\left\langle U^{\dagger}(t) I(t) U(t)\right\rangle_{0}=I_{1}+I_{2}+I_{3}+\boldsymbol{O}\left(\mathcal{T}^{3}\right),
$$

where

$$
\begin{aligned}
I_{1}(t)= & -i \int_{-\infty}^{t} d t^{\prime}\left\langle\left[I_{T}(t), H_{T}\left(t^{\prime}\right)\right]\right\rangle_{0}, \\
I_{2}(t)= & \int_{-\infty}^{t} d t_{1} \int_{-\infty}^{t} d t_{2}\left\langle H_{T}\left(t_{1}\right) I_{d}(t) H_{T}\left(t_{2}\right)\right\rangle_{0}, \\
I_{3}(t)= & -\int_{-\infty}^{t} d t_{1} \int_{-\infty}^{t_{1}} d t_{2}\left[\left\langle H_{T}\left(t_{1}\right) H_{T}\left(t_{2}\right) I_{d}(t)\right\rangle_{0}\right. \\
& \left.+\left\langle I_{d}(t) H_{T}\left(t_{1}\right) H_{T}\left(t_{2}\right)\right\rangle_{0}\right] .
\end{aligned}
$$

In these equations, $\langle\ldots\rangle_{0}$ indicates expectation with respect to the state without the rf perturbation. In the homogeneous case, only $I_{1}(t)$ takes a nonzero value.

We will assume that the initial density of nonpairing state$|2\rangle$ atoms is equal to zero; it is straightforward to treat the more general case, but the result is rather cumbersome. In this case, the total current is positive definite, while $I_{3}(t)$ in Eq. $(5.11 \mathrm{c})$ vanishes exactly. For the finite sample geometry of interest here, both $I_{1}(t)$ and $I_{2}(t)$ are comparable and must be evaluated, e.g., $I_{1}(t)$ can take negative values for a system with an edge. We find the following result for the time-averaged local current at $x=x_{0}$ :

$$
\begin{aligned}
I\left(x_{0}, \omega\right)= & 2 \pi \mathcal{T}^{2} \int_{k_{y}} \sum_{p, k}\left\{\frac{1}{2}\left[1+\gamma_{k_{y}}(p)\right]\left|w\left(k, x_{0}\right)\right|^{2}\right. \\
& \times\left|\int_{x} u_{k_{y}}(p, x) w^{*}(k, x)\right|^{2} \delta\left[\omega+E_{k_{y}}(p)-\xi_{k_{y}}(k)\right] \\
& +\frac{1}{2}\left[1-\gamma_{k_{y}}(p)\right]\left|w\left(k, x_{0}\right)\right|^{2}\left|\int_{x} v_{k_{y}}(p, x) w^{*}(k, x)\right|^{2} \\
& \left.\times \delta\left[\omega-E_{k_{y}}(p)-\xi_{k_{y}}(k)\right]\right\} .
\end{aligned}
$$

In this equation, $\gamma_{k_{y}}(p)=2\left\langle a_{k_{y}}^{\dagger}(p) a_{k_{y}}(p)\right\rangle-1$ encodes the occupation of the states [induced by the quench; cf. Eq. (2.9)].

In the derivation of the local current, we did not use the explicit form of $u_{k_{y}}(p, x), v_{k_{y}}(p, x)$, or $w(k, x)$, but only the assumption that each is either purely real or imaginary. This assumption should be true for any realistic boundary conditions given the form of paired and nonpairing Hamiltonians (5.1) and (5.5). Equation (5.12) therefore applies to any semi-infinite or infinite system governed by Eq. (5.1).

Equation (5.12) takes the form of Fermi's golden rule. The first (second) term is due to the process where the excited(ground-) state Cooper pair with energy $E_{k_{y}}(p)\left[-E_{k_{y}}(p)\right]$ absorbs a rf photon, producing a state- $|2\rangle$ atom with energy $\xi_{k_{y}}(k)$ and an unpaired state- $|1\rangle$ atom with energy zero. The factor $\frac{1}{2}\left[1+\gamma_{k_{y}}(p)\right]\left\{\frac{1}{2}\left[1-\gamma_{k_{y}}(p)\right]\right\}$ encodes the probability that the quasiparticle state $\left\{p, k_{y}\right\}$ is initially occupied (unoccupied) before the application of rf radiation. The factor $\left|w\left(k, x_{0}\right)\right|^{2}$ in each term is the probability that an atom exists at $x=x_{0}$ in the nonpairing $|2\rangle$ state with momenta $\left\{k, k_{y}\right\}$. 
Although Eq. (5.12) is very similar to the homogeneous result [e.g., Eq. (2.9) with only $\tilde{u}_{0, \mathbf{k}}, \tilde{v}_{0, \mathbf{k}}$ nonzero], it has very different implications for bulk versus edge states. The transition rates are proportional to the squared overlaps

$$
\begin{aligned}
& T_{u ; k_{y}}(p, k) \equiv\left|\int_{x} u_{k_{y}}(p, x) w^{*}(k, x)\right|^{2}, \\
& T_{v ; k_{y}}(p, k) \equiv\left|\int_{x} v_{k_{y}}(p, x) w^{*}(k, x)\right|^{2} .
\end{aligned}
$$

When coherence factors $u_{k_{y}}(p, x)$ and $v_{k_{y}}(p, x)$ describe extended bulk states, then the product of these with extended (box) wave function $w^{*}(k, x)$ is of order $1 / \mathcal{L}$, where $\mathcal{L}$ is the extension of the system in the $x$ direction. For $p=k$, the integral over $x$ gives a factor of $\mathcal{L}$, so that $T_{u, v ; k_{y}}$ are of order unity. When $u_{k_{y}}(p, x)$ and $v_{k_{v}}(p, x)$ describe a spatially localized edge mode, these are a factor of $1 / \mathcal{L}$ smaller. We conclude that the contribution of the edge states is suppressed by a factor of the linear system size. Moreover, because $k_{y}$ runs over a finite range for the edge modes, the edge signal is delocalized throughout the bulk of the sample [since it obtains from integrating $\left|w\left(k, x_{0}\right)\right|^{2}$ over a finite range of $k$ for which energy conservation is satisfied].

Thus, local rf spectroscopy of this type is not well suited to detect Majorana edge modes.

\section{Tunneling}

By contrast, the local tunneling conductance is given by

$$
\begin{aligned}
G\left(x_{0}, \tilde{V}\right)= & 2 \pi \mathcal{T}^{2} v_{0} \int_{k_{y}} \sum_{p}\left\{\left|u_{k_{y}}\left(p, x_{0}\right)\right|^{2} \delta\left[\tilde{V}+E_{k_{y}}(p)\right]\right. \\
& \left.+\left|v_{k_{y}}\left(p, x_{0}\right)\right|^{2} \delta\left[\tilde{V}-E_{k_{y}}(p)\right]\right\} .
\end{aligned}
$$

This is independent of the distribution function (quenchinduced occupancy), and gives equal weight to discrete bound states (edge modes) and continuum bulk modes. The contribution of each of the latter is suppressed by a factor of $1 / \mathcal{L}$, but this is compensated by the summation over $p$.

When the metal tip is placed deep in the bulk, the tunneling spectrum resembles that of the homogeneous case. Near the boundary, signal within the gap is contributed only by the edge modes.

\section{CONCLUSION}

In this paper, we have obtained the rf and tunneling spectra of a quench-induced out-of-equilibrium steady state. In particular, we focused on the Floquet system with timeperiodic order parameter induced by a phase III quench. One important aspect of our result is the connection between these experimental observables and the distribution function. We found that the distribution function plays an essential role in the bulk rf spectrum. We demonstrated that the reason the timeaveraged rf (but not tunneling) spectra are in good agreement with static quasiequilibrium (phase II) approximations lies in the distribution function, which is forced to exhibit a population inversion in the basis of Floquet states due to the conservation of the topological pseudospin winding number. This crucial information is missing in the tunneling spectrum, and leads to the disappearance of the gap in tunneling spectra for strong phase III quenches characterized by large amplitude oscillations in the order parameter. Finally, we showed that local rf is not a good method to detect Majorana edge states, due to the nonlocal character of the radiation-induced transitions to nonpairing states.

\section{ACKNOWLEDGMENTS}

We thank L. D'Alessio, V. Gurarie, K. Hazzard, and E. Yuzbashyan for useful discussions. We acknowledge funding from the Welch Foundation under Grant No. C-1809 and from an Alfred P. Sloan Research Fellowship (No. BR2014-035).

\section{APPENDIX A: RF AND TUNNELING AMPLITUDE HARMONICS}

In the superfluid Floquet phase, the rf current given by Eq. (2.7) exhibits modulations at harmonics of the drive frequency $\Omega$. These are encoded in

$$
\begin{aligned}
I_{\mathrm{rf}}(p)= & \frac{\pi \mathcal{T}^{2}}{2} \sum_{n, \mathbf{k}}\left\{\left[\left(1-\gamma_{\mathbf{k}}\right)\left(1-f_{\mathbf{k}}^{(d)}\right)-\left(1+\gamma_{\mathbf{k}}\right) f_{\mathbf{k}}^{(d)}\right]\left(\tilde{v}_{n, \mathbf{k}}^{*} \tilde{v}_{n+p, \mathbf{k}}+\tilde{v}_{n-p, \mathbf{k}}^{*} \tilde{v}_{n, \mathbf{k}}\right) \delta\left(\omega-\xi_{\mathbf{k}}-E_{\mathbf{k}}^{(F)}+n \Omega\right)\right. \\
& \left.+\left[\left(1+\gamma_{\mathbf{k}}\right)\left(1-f_{\mathbf{k}}^{(d)}\right)-\left(1-\gamma_{\mathbf{k}}\right) f_{\mathbf{k}}^{(d)}\right]\left(\tilde{u}_{n, \mathbf{k}}^{*} \tilde{u}_{n+p, \mathbf{k}}+\tilde{u}_{n-p, \mathbf{k}}^{*} \tilde{u}_{n, \mathbf{k}}\right) \delta\left(\omega-\xi_{\mathbf{k}}+E_{\mathbf{k}}^{(F)}-n \Omega\right)\right\} \\
& -\frac{i \mathcal{T}^{2}}{2} \sum_{n, \mathbf{k}}\left\{\left[\left(1-\gamma_{\mathbf{k}}\right)\left(1-f_{\mathbf{k}}^{(d)}\right)-\left(1+\gamma_{\mathbf{k}}\right) f_{\mathbf{k}}^{(d)}\right]\left(\tilde{v}_{n, \mathbf{k}}^{*} \tilde{v}_{n+p, \mathbf{k}}-\tilde{v}_{n-p, \mathbf{k}}^{*} \tilde{v}_{n, \mathbf{k}}\right) \frac{1}{\omega-\xi_{\mathbf{k}}-E_{\mathbf{k}}^{(F)}+n \Omega}\right. \\
& \left.-\left[\left(1+\gamma_{\mathbf{k}}\right)\left(1-f_{\mathbf{k}}^{(d)}\right)-\left(1-\gamma_{\mathbf{k}}\right) f_{\mathbf{k}}^{(d)}\right]\left(\tilde{u}_{n, \mathbf{k}}^{*} \tilde{u}_{n+p, \mathbf{k}}-\tilde{u}_{n-p, \mathbf{k}}^{*} \tilde{u}_{n, \mathbf{k}}\right) \frac{1}{\omega-\xi_{\mathbf{k}}+E_{\mathbf{k}}^{(F)}-n \Omega}\right\} .
\end{aligned}
$$

An analogous expression for harmonics of the tunneling conductance is given by

$$
\begin{aligned}
G(p)= & \pi \mathcal{T}^{2} v_{0} \sum_{n, \mathbf{k}}\left[\left(\tilde{v}_{n, \mathbf{k}}^{*} \tilde{v}_{n+p, \mathbf{k}}+\tilde{v}_{n-p, \mathbf{k}}^{*} \tilde{v}_{n, \mathbf{k}}\right) \delta\left(\tilde{V}-E_{\mathbf{k}}^{(F)}+n \Omega\right)+\left(\tilde{u}_{n, \mathbf{k}}^{*} \tilde{u}_{n+p, \mathbf{k}}+\tilde{u}_{n-p, \mathbf{k}}^{*} \tilde{u}_{n, \mathbf{k}}\right) \delta\left(\tilde{V}+E_{\mathbf{k}}^{(F)}-n \Omega\right)\right] \\
& -i \mathcal{T}^{2} v_{0} \sum_{n, \mathbf{k}}\left[\left(\tilde{v}_{n, \mathbf{k}}^{*} \tilde{v}_{n+p, \mathbf{k}}-\tilde{v}_{n-p, \mathbf{k}}^{*} \tilde{v}_{n, \mathbf{k}}\right) \frac{1}{\tilde{V}-E_{\mathbf{k}}^{(F)}+n \Omega}-\left(\tilde{u}_{n, \mathbf{k}}^{*} \tilde{u}_{n+p, \mathbf{k}}-\tilde{u}_{n-p, \mathbf{k}}^{*} \tilde{u}_{n, \mathbf{k}}\right) \frac{1}{\tilde{V}+E_{\mathbf{k}}^{(F)}-n \Omega}\right] .
\end{aligned}
$$


We find that $\tilde{u}_{n, \mathbf{k}}, \tilde{v}_{n, \mathbf{k}}$ are real, so the second term in Eqs. (A1) and (A2) can be ignored if only the real parts of $I_{\mathrm{rf}}(p)$ and $G(p)$ are needed.

\section{APPENDIX B: FLOQUET STATES AND OCCUPATIONS VIA INTEGRABILITY: EXPLICIT SOLUTION IN PHASE III}

Through self-consistent mean field theory and the use of the Lax construction, the order parameter $\Delta(t)$ as a function of time is determined $\left(\Delta \equiv \sqrt{\mathrm{R}} e^{-i \phi}\right)$. The amplitude $(\sqrt{\mathrm{R}})$ and the argument $(-\phi)$ of the complex order parameter follow the EOM [9]

$$
\begin{gathered}
\dot{\mathrm{R}}^{2}=\left(\mathrm{R}_{+}-\mathrm{R}\right)\left(\mathrm{R}-\mathrm{R}_{-}\right)\left(\mathrm{R}+\widetilde{\mathrm{R}}_{+}\right)\left(\mathrm{R}+\widetilde{\mathrm{R}}_{-}\right), \\
\dot{\phi}=\frac{3}{2} \mathrm{R}+2 \mathrm{~m}-\frac{\psi}{\mathrm{R}},
\end{gathered}
$$

where

$$
\begin{gathered}
\mathrm{R}_{ \pm} \equiv\left(\Delta_{1} \pm \Delta_{2}\right)^{2}, \\
\widetilde{\mathrm{R}}_{ \pm} \equiv\left(\sqrt{2 \mu_{1}-\Delta_{1}^{2}} \pm \sqrt{2 \mu_{2}-\Delta_{2}^{2}}\right)^{2}, \\
\mathrm{~m} \equiv \frac{1}{2}\left(\mu_{1}+\mu_{2}-\Delta_{1}^{2}-\Delta_{2}^{2}\right), \\
\psi \equiv \frac{1}{2}\left(\Delta_{1}^{2}-\Delta_{2}^{2}\right)\left(\Delta_{1}^{2}-\Delta_{2}^{2}-2 \mu_{1}+2 \mu_{2}\right) .
\end{gathered}
$$

The parameters $\Delta_{1,2}$ and $\mu_{1,2}$ are determined via Eq. (4.1) by the two pairs of isolated roots that characterize a phase III quench. For a given quench specified by the coordinates $\left\{\Delta_{0}^{(i)}, \Delta_{0}^{(f)}\right\}$, these roots solve a certain transcendental equation [9]. Results can be obtained numerically for a given fixed particle density $n$ and ultraviolet energy cutoff $\Lambda$.

The solution $\phi(t)$ to Eq. (B2) is a combination of a periodic part $\Phi(t)$ and linear part $2 \mu_{\infty} t$. Here, $\Phi(t)$ shares the same period $T$ with $\mathrm{R}(t)$, and $\mu_{\infty}$ is defined as

$$
\mu_{\infty} \equiv \frac{1}{2 T} \int_{0}^{T} d t \dot{\phi}=\frac{1}{2 T} \int_{0}^{T} d t\left[\frac{3}{2} \mathrm{R}(t)+2 \mathrm{~m}-\frac{\psi}{\mathrm{R}(t)}\right] .
$$

\section{Lax reduced solution}

In the following, a "Lax reduced" solution [9] to the BCS spin dynamics is utilized to find the explicit solution to our problem, i.e., the asymptotic steady state following a sudden quench of coupling strength. We consider a reduced solution with the same order parameter (isolated roots) as a particular phase III quench, but with different initial conditions. The existence of such solutions, along with an explicit prescription for constructing them, was detailed in Secs. III C and IV B of [9], based upon previous $s$-wave work [29]. In terms of Anderson pseudospins, the reduced solution can be written as a product of a sign function $\zeta(\varepsilon)$ and a pseudospin function $\vec{s}_{0}(\varepsilon)$,

$$
\vec{s}_{R}(\varepsilon)=\zeta(\varepsilon) \vec{s}_{0}(\varepsilon),
$$

where $\zeta(\varepsilon)= \pm 1$. The single-particle levels are labeled via the momentum squared $\varepsilon \equiv k^{2}$. To qualify as a solution, $\zeta(\varepsilon)$ will exhibit a discontinuous jump at some $\varepsilon$ from +1 to -1 ; this is closely related to the soliton solution discussed above in Sec. IV C. The function $\vec{s}_{0}(\varepsilon)$ depends only on the order parameter, and is given by

$$
\begin{aligned}
s_{0}^{z}(\varepsilon) & =a(\varepsilon) \mathrm{R}+b(\varepsilon), \\
s_{0}^{-}(\varepsilon) & =\frac{a(\varepsilon)}{2 \sqrt{\varepsilon \mathrm{R}}}\left[-i \dot{\mathrm{R}}+\mathrm{R}^{2}+(4 \mathrm{~m}-2 \varepsilon) \mathrm{R}+2 \psi\right] e^{-i \phi},
\end{aligned}
$$

where $a(\varepsilon)$ and $b(\varepsilon)$ are functions of $\varepsilon$ :

$$
\begin{aligned}
& a(\varepsilon)=\frac{\varepsilon}{4 E_{1}(\varepsilon) E_{2}(\varepsilon)}, \\
& b(\varepsilon)=-\frac{E_{1}^{2}(\varepsilon)+E_{2}^{2}(\varepsilon)-\left(\mu_{1}-\mu_{2}\right)^{2}}{4 E_{1}(\varepsilon) E_{2}(\varepsilon)} .
\end{aligned}
$$

Here, $E_{a}(\varepsilon) \equiv \sqrt{\left(\varepsilon / 2-\mu_{a}\right)^{2}+\Delta_{a}^{2} \varepsilon}, a=1,2$, is a $p+i p$ quasiparticle energy [Eq. (1.3)].

The order parameter is defined self-consistently by

$$
\Delta(t) \equiv-G \sum_{i} \sqrt{\varepsilon_{i}} s_{i}^{-} .
$$

Substituting $s_{i}^{-}$in this equation from the reduced solution in Eq. (B6) and using Eq. (B7), we find that the left-hand side reduces to

$$
\begin{aligned}
& -G\left(\sum_{i} \zeta_{i} a_{i}\right) \frac{-i \dot{\mathrm{R}}+\mathrm{R}^{2}+4 \mathrm{mR}+2 \psi}{2 \sqrt{\mathrm{R}}} e^{-i \phi} \\
& +G\left(\sum_{i} \zeta_{i} \varepsilon_{i} a_{i}\right) \sqrt{\mathrm{R}} e^{-i \phi}
\end{aligned}
$$

This gives $\sqrt{\mathrm{R}} e^{-i \phi}$ if and only if

$$
\sum_{i} \zeta_{i} a_{i}=0, \quad \sum_{i} \zeta_{i} \varepsilon_{i} a_{i}=\frac{1}{G},
$$

which serve as the constraints for the Lax reduced solution. Under such constraints, we find the total $z$ spin $J$ (related to the fixed number of particles $N$ ) and the total energy $E$ are indeed conserved quantities since

$$
\begin{aligned}
J & \equiv \sum_{i} \zeta_{i} s_{0, i}^{z}=\sum_{i} \zeta_{i} b_{i}, \\
E & \equiv \sum_{i} \zeta_{i} \varepsilon_{i} s_{0, i}^{z}-\frac{\mathrm{R}}{G}=\sum_{i} \zeta_{i} \varepsilon_{i} b_{i} .
\end{aligned}
$$

Another important quantity is the Lax norm [9], which generates all the integrals of motion. We find that the Lax norm of the reduced solution is given by

$$
L_{2}(u)=\left[\sum_{i} \frac{\zeta_{i} \varepsilon_{i}}{2 \sqrt{\mathcal{Q}_{4}\left(\varepsilon_{i}\right)}} \frac{1}{\varepsilon_{i}-u}\right]^{2} \mathcal{Q}_{4}(u) .
$$

The $\left\{\zeta_{i}\right\}$ can in principle be determined by equating Eq. (B13) to its initial value.

To find the spinor of $\vec{s}_{0}$ parametrized as

$$
\left[\begin{array}{c}
u_{0}(\varepsilon, t) \\
v_{0}(\varepsilon, t)
\end{array}\right]=\left[\begin{array}{l}
\left|u_{0}(\varepsilon, t)\right| e^{-i \theta_{u}(\varepsilon, t)} \\
\left|v_{0}(\varepsilon, t)\right| e^{-i \theta_{v}(\varepsilon, t)}
\end{array}\right],
$$


we use

$$
\begin{aligned}
s_{0}^{-}(\varepsilon, t) & =u_{0}^{*}(\varepsilon, t) v_{0}(\varepsilon, t), \\
s_{0}^{z}(\varepsilon, t) & =\frac{1}{2}\left[\left|v_{0}(\varepsilon, t)\right|^{2}-\left|u_{0}(\varepsilon, t)\right|^{2}\right] .
\end{aligned}
$$

In the rotating frame $s^{-} \rightarrow s^{-} e^{i \phi}$, this yields

$$
\begin{gathered}
\left|u_{0}(\varepsilon, t)\right|=\sqrt{\frac{1}{2}-[a(\varepsilon) \mathrm{R}(t)+b(\varepsilon)]}, \\
\left|v_{0}(\varepsilon, t)\right|=\sqrt{\frac{1}{2}+[a(\varepsilon) \mathrm{R}(t)+b(\varepsilon)]} \\
\theta_{u}(\varepsilon, t)-\theta_{v}(\varepsilon, t)=\arg \left(-i \dot{\mathrm{R}}+\mathrm{R}^{2}+4 \mathrm{mR}+2 \psi-2 \varepsilon \mathrm{R}\right) .
\end{gathered}
$$

Inserting Eq. (B16) into (1.7), we get

$$
\begin{gathered}
\dot{\theta}_{u}=-\frac{\varepsilon}{2}+\frac{1}{2} \frac{d \phi}{d t}+\frac{a\left(\mathrm{R}^{2}+4 \mathrm{mR}+2 \psi-2 \varepsilon \mathrm{R}\right)}{1-2(a \mathrm{R}+b)}, \\
\dot{\theta}_{v}=\frac{\varepsilon}{2}-\frac{1}{2} \frac{d \phi}{d t}+\frac{a\left(\mathrm{R}^{2}+4 \mathrm{mR}+2 \psi-2 \varepsilon \mathrm{R}\right)}{1+2(a \mathrm{R}+b)} .
\end{gathered}
$$

We define $\varepsilon_{+}$and $\varepsilon_{-}$by

$$
\varepsilon_{ \pm} \equiv \frac{\mathrm{R}_{ \pm}^{2}+4 \mathrm{mR}_{ \pm}+2 \psi}{2 \mathrm{R}_{ \pm}}
$$

Equation (B18) [Eq. (B19)] is valid as long as $\varepsilon \neq \varepsilon_{+}$or $t \neq 0$ $\left(\varepsilon \neq \varepsilon_{-}\right.$or $\left.t \neq \frac{T}{2}\right) . \theta_{u}\left(\varepsilon_{+}, 0\right)$ and $\theta_{v}\left(\varepsilon_{-}, \frac{T}{2}\right)$ are unimportant since $\left|u\left(\varepsilon_{+}, 0\right)\right|=0$ and $\left|v\left(\varepsilon_{-}, \frac{T}{2}\right)\right|=0$.

We next switch from the frame rotating with phase $\phi(t)=$ $2 \mu_{\infty} t+\Phi(t)$ to that rotating only with its linear piece $2 \mu_{\infty} t$. This is equivalent to replacing $d \phi / d t \rightarrow 2 \mu_{\infty}$ in Eqs. (B18) and (B19). The resulting equations depend on $t$ only through $\mathrm{R}$, which implies that $\theta_{u}\left(\theta_{v}\right)$ should be a sum of a time-periodic function and a linear piece $-E_{u} t\left(-E_{v} t\right)$. The intercept $E_{u}$ $\left(E_{v}\right)$ is determined by integrating Eq. (B18) [Eq. (B19)] over one period,

$$
\begin{aligned}
E_{u}(\varepsilon) & =-\frac{1}{T} \int_{0}^{T} d t \dot{\theta}_{u}(\varepsilon, t) \\
& =\frac{\varepsilon}{2}-\mu_{\infty}-\frac{1}{T} \int_{0}^{T} d t f_{-}(\varepsilon, t), \\
E_{v}(\varepsilon) & =-\frac{1}{T} \int_{0}^{T} d t \dot{\theta}_{v}(\varepsilon, t) \\
& =-\frac{\varepsilon}{2}+\mu_{\infty}-\frac{1}{T} \int_{0}^{T} d t f_{+}(\varepsilon, t) .
\end{aligned}
$$

Here,

$$
f_{ \pm}(\varepsilon, t) \equiv \frac{a(\varepsilon)\left[\mathrm{R}^{2}(t)+4 \mathrm{mR}(t)+2 \psi-2 \varepsilon \mathrm{R}(t)\right]}{1 \pm 2[a(\varepsilon) \mathrm{R}(t)+b(\varepsilon)]} .
$$

We find that $E_{v}(\varepsilon)-E_{u}(\varepsilon)=-n_{0}(\varepsilon) \Omega$ from Eq. (B17), where $n_{0}(\varepsilon)=\theta\left(\varepsilon-\varepsilon_{+}\right) \theta\left(\varepsilon_{-}-\varepsilon\right)$. As a result, $\left\{u_{0}(\varepsilon, t)\right.$, $\left.v_{0}(\varepsilon, t)\right\}$ is a Floquet state and can be parametrized as $\left\{\left|u_{0}(\varepsilon, t)\right| e^{-i \Theta_{u}(\varepsilon, t)},\left|v_{0}(\varepsilon, t)\right| e^{-i \Theta_{v}(\varepsilon, t)}\right\} e^{+i E_{0}(\varepsilon) t}$. Here, the quasienergy $E_{0} \equiv E_{u}(\bmod \Omega), \Theta_{u}(t+T) \equiv \Theta_{u}(t)(\bmod 2 \pi)$, and $\Theta_{v}(t+T) \equiv \Theta_{v}(t)(\bmod 2 \pi) . \Theta_{u}, \Theta_{v}$ can be obtained by integrating Eqs. (B18) and (B19). Assuming $\mathrm{R}(t=0)=\mathrm{R}_{+}$ and $\Phi(t=0)=0$, we deduce the integration constant from Eq. (B17):

$$
\begin{aligned}
\Theta_{u}(\varepsilon, 0)-\Theta_{v}(\varepsilon, 0) & =\pi \theta\left(\varepsilon-\varepsilon_{+}\right), \\
\Theta_{u}\left(\varepsilon, \frac{T}{2}\right)-\Theta_{v}\left(\varepsilon, \frac{T}{2}\right) & =\pi \theta\left(\varepsilon-\varepsilon_{-}\right) .
\end{aligned}
$$

Then, we obtain

$$
\begin{aligned}
\Theta_{u}(\varepsilon, t)= & \int_{0}^{t} d t^{\prime} f_{-}\left(\varepsilon, t^{\prime}\right)+\left[-\frac{\varepsilon}{2}+\mu_{\infty}+E_{0}(\varepsilon)\right] t \\
\Theta_{v}(\varepsilon, t)= & -\pi \theta\left(\varepsilon-\varepsilon_{+}\right)+\int_{0}^{t} d t^{\prime} f_{+}\left(\varepsilon, t^{\prime}\right) \\
& +\left[\frac{\varepsilon}{2}-\mu_{\infty}+E_{0}(\varepsilon)\right] t .
\end{aligned}
$$

In the scheme where we take the quasienergy $E_{0}=E_{u}$, we have $\Theta_{u}(\varepsilon, t+T)=\Theta_{u}(\varepsilon, t), \Theta_{v}(\varepsilon, t+T)=\Theta_{v}(\varepsilon, t)+$ $2 \pi n_{0}(\varepsilon)$, and Eq. (B24) simplifies to

$$
\begin{aligned}
\Theta_{u}(\varepsilon, t)= & {\left[\int_{0}^{t} d t^{\prime}-\frac{t}{T} \int_{0}^{T} d t^{\prime}\right] f_{-}\left(\varepsilon, t^{\prime}\right) } \\
\Theta_{v}(\varepsilon, t)= & {\left[\int_{0}^{t} d t^{\prime}-\frac{t}{T} \int_{0}^{T} d t^{\prime}\right] f_{+}\left(\varepsilon, t^{\prime}\right)-\pi \theta\left(\varepsilon-\varepsilon_{+}\right) } \\
& +2 \pi \theta\left(\varepsilon-\varepsilon_{+}\right) \theta\left(\varepsilon_{-}-\varepsilon\right) \frac{t}{T}
\end{aligned}
$$

Combining Eqs. (B16) and (B24), we obtain the complete expression for $\left[u_{0}(\varepsilon, t) \quad v_{0}(\varepsilon, t)\right]^{T}$. Then, the spinor of the Lax reduced solution can be written as

$$
\begin{aligned}
{\left[\begin{array}{c}
u(\varepsilon, t) \\
v(\varepsilon, t)
\end{array}\right]=} & \sqrt{\frac{1+\zeta(\varepsilon)}{2}}\left[\begin{array}{l}
\left|u_{0}(\varepsilon, t)\right| e^{-i \Theta_{u}(\varepsilon, t)} \\
\left|v_{0}(\varepsilon, t)\right| e^{-i \Theta_{v}(\varepsilon, t)}
\end{array}\right] e^{+i E_{0}(\varepsilon) t} \\
& +\sqrt{\frac{1-\zeta(\varepsilon)}{2}}\left[\begin{array}{c}
\left|v_{0}(\varepsilon, t)\right| e^{i \Theta_{v}(\varepsilon, t)} \\
-\left|u_{0}(\varepsilon, t)\right| e^{i \Theta_{u}(\varepsilon, t)}
\end{array}\right] e^{-i E_{0}(\varepsilon) t+i \Gamma(\varepsilon)} .
\end{aligned}
$$

Here, $\Gamma(\varepsilon)$ is some time-independent phase.

\section{Initial condition}

We assume that the general solution to the self-consistent $\mathrm{BdG}$ equations following a quench takes a similar form

$$
\begin{aligned}
{\left[\begin{array}{c}
u(\varepsilon, t) \\
v(\varepsilon, t)
\end{array}\right]=} & \sqrt{\frac{1-\gamma(\varepsilon)}{2}\left[\begin{array}{l}
\left|u_{0}(\varepsilon, t)\right| e^{-i \Theta_{u}(\varepsilon, t)} \\
\left|v_{0}(\varepsilon, t)\right| e^{-i \Theta_{v}(\varepsilon, t)}
\end{array}\right] e^{+i E_{0}(\varepsilon) t}} \\
& +\sqrt{\frac{1+\gamma(\varepsilon)}{2}}\left[\begin{array}{c}
\left|v_{0}(\varepsilon, t)\right| e^{i \Theta_{v}(\varepsilon, t)} \\
-\left|u_{0}(\varepsilon, t)\right| e^{i \Theta_{u}(\varepsilon, t)}
\end{array}\right] e^{-i E_{0}(\varepsilon) t+i \Gamma(\varepsilon)}
\end{aligned}
$$

The Lax reduced solution in Eq. (B26) is an extreme example where $\gamma=-\zeta$ only takes values equal to \pm 1 . In order to solve the self-consistent conditions in Eq. (B11), $\zeta(\varepsilon)$ must exhibit a discontinuity ("Fermi step"). The reduced solution therefore cannot apply to a quench from a BCS initial state since the latter initially has a smooth pseudospin texture in momentum space, and the BCS dynamics does not change this. The solution for the quench involves replacing $-\zeta(\varepsilon)$ with a smooth $\gamma(\varepsilon)$ that nevertheless winds from +1 to -1 with increasing $\varepsilon$.

The conservation of energy $E, z$-component angular momentum $J$ (particle number), and the Lax norm allows us 
to determine the $\gamma_{i}$ for the post-quench state. Substituting Eq. (B27) into (B15), we have

$$
\begin{aligned}
& s_{i}^{-}(t)=-\gamma_{i} u_{0, i}^{*} v_{0, i} \\
&+\frac{\sqrt{1-\gamma_{i}^{2}}}{2}\left(-e^{i \Gamma_{i}} u_{0, i}^{* 2} e^{-2 i E_{i} t}+e^{-i \Gamma_{i}} v_{0, i}^{2} e^{2 i E_{i} t}\right), \\
& s_{i}^{z}(t)=-\gamma_{i} \frac{1}{2}\left[\left|v_{0, i}(t)\right|^{2}-\left|u_{0, i}(t)\right|^{2}\right] \\
&+\frac{\sqrt{1-\gamma_{i}^{2}}}{2}\left(-e^{-i \Gamma_{i}} u_{0, i} v_{0, i} e^{2 i E_{i} t}\right. \\
&\left.-e^{i \Gamma_{i}} u_{0, i}^{*} v_{0, i}^{*} e^{-2 i E_{i} t}\right)
\end{aligned}
$$

These can be considered as a combination of $\vec{s}_{0, i}(t)$ and an oscillating spin with energy-dependent frequency $\vec{s}_{\text {osc,i }}(t)$, weighted by $-\gamma_{i}$ and $\sqrt{1-\gamma_{i}^{2}}$ separately, i.e., $\vec{s}_{i}(t)=$ $-\gamma_{i} \vec{s}_{0, i}+\sqrt{1-\gamma_{i}^{2}} \vec{s}_{\mathrm{osc}, \mathrm{i}}$. As $t \rightarrow \infty$, through repeated integration by parts or the saddle-point approximation, we find that the contribution of $\vec{s}_{\text {osc,i }}$ to $E, J$, and the Lax norm vanishes. Therefore, all of the conserved quantities can be obtained by replacing $\zeta$ with $-\gamma$ in Eqs. (B11)-(B13), which leads to

$$
\begin{array}{ll}
\sum_{i} \gamma_{i} a_{i}=0, \quad \sum_{i} \gamma_{i} \varepsilon_{i} a_{i}=-\frac{1}{G}, \\
\sum_{i} \gamma_{i} b_{i}=-J, \quad \sum_{i} \gamma_{i} \varepsilon_{i} b_{i}=-E,
\end{array}
$$

and

$$
L_{2}(u)=\left[\sum_{j} \frac{\gamma_{j} \varepsilon_{j}}{2 \sqrt{\mathcal{Q}_{4}\left(\varepsilon_{j}\right)}} \frac{1}{\varepsilon_{j}-u}\right]^{2} \mathcal{Q}_{4}(u) .
$$

We now let $u$ in Eq. (B30) approach the positive real axis from above and below, $u \rightarrow u \pm i \eta, u \in \mathbb{R}^{+}$and $\eta \rightarrow 0$. From the residue theorem,

$$
\begin{aligned}
L_{2}(u \pm i \eta)= & v^{2}\left[P \int_{0}^{\varepsilon_{\Lambda}} d \varepsilon \frac{\gamma(\varepsilon) \varepsilon}{2 \sqrt{\mathcal{Q}_{4}(\varepsilon)}} \frac{1}{\varepsilon-u}\right. \\
& \left. \pm i \pi \frac{\gamma(u) u}{2 \sqrt{\mathcal{Q}_{4}(u)}}\right]^{2} \mathcal{Q}_{4}(u),
\end{aligned}
$$

where $v \equiv \mathcal{L}^{2} / 8 \pi$ is the bare density of states, and $\mathcal{L}$ denotes the linear system size. This is equal to the Lax norm of the pre-quench state

$$
L_{2}(u \pm i \eta)=\frac{v^{2}}{4} I_{\mp}(u)
$$

which gives

$$
|\gamma(\varepsilon)|=\frac{\left|\sqrt{I_{+}(\varepsilon)}-\sqrt{I_{-}(\varepsilon)}\right|}{2 \pi \varepsilon} .
$$

This shares the same form as the pseudospin distribution function in phases I and II. Here, $I_{+}(\varepsilon)$ is a certain function that depends upon the parameters of the pre-quench state, as well as the strength and direction of the quench (i.e., the quench coordinates $\left.\left\{\Delta_{0}^{(i)}, \Delta_{0}^{(f)}\right\}\right)$, while $I_{-}(\varepsilon)$ is its complex conjugate; an explicit expression appears in [9]. The sign of $\gamma$ is determined by enforcing continuity whenever $\gamma(\varepsilon) \rightarrow 0$ with a nonzero slope [9]. We find that $\gamma(\varepsilon)$ winds exactly once from +1 at small $\varepsilon$ to -1 at large $\varepsilon$ for all quenches in phase III; see, e.g., Fig. 9. For all phase III quenches that we have investigated including " $A$ " and " $B$ " indicated in Fig. 1, we have used the result for $\gamma(\varepsilon)$ and verified that all four relations in Eq. (B29) hold.

We find that $\Gamma(\varepsilon)$ in Eq. (B27) must be 0 or $\pi$ due to the invariance of the pseudospin equations of motion under the effective time-reversal transformation [9]

$$
\begin{aligned}
s^{z}(\varepsilon, t) & \rightarrow s^{z}(\varepsilon,-t), \\
s^{ \pm}(\varepsilon, t) & \rightarrow s^{\mp}(\varepsilon,-t), \\
\Delta(t) & \rightarrow \Delta^{*}(-t) .
\end{aligned}
$$

\section{Phases II, III border}

On the border of phases II and III, $\Delta_{1}$ approaches 0 , and the time evolution of the modulus and phase of the order parameter is given by

$$
\begin{aligned}
\sqrt{\mathrm{R}(t)} & =\Delta_{2} \quad \text { (const), } \\
\phi(t) & =2 \mu_{2} t .
\end{aligned}
$$

The Lax reduced solution on the border can be obtained from Eqs. (B34), (B7), and (B8):

$$
\begin{aligned}
s^{z}(\varepsilon) & =-\zeta(\varepsilon) \operatorname{sgn}\left(\varepsilon-2 \mu_{1}\right) \frac{\left(\frac{\varepsilon}{2}-\mu_{2}\right)}{2 E_{2}(\varepsilon)}, \\
s^{-}(\varepsilon) & =-\zeta(\varepsilon) \operatorname{sgn}\left(\varepsilon-2 \mu_{1}\right) \frac{\sqrt{\varepsilon} \Delta_{2}}{2 E_{2}(\varepsilon)} e^{-2 i \mu_{2} t} .
\end{aligned}
$$

These are the pseudospin configurations for ground- (excited-) state Cooper pairs when $\zeta(\varepsilon) \operatorname{sgn}\left(\varepsilon-2 \mu_{1}\right)=1(-1)$.

In addition, following all the equations for the spinor in phase III, we find

$$
\begin{aligned}
E_{0}(\varepsilon) & =\operatorname{sgn}\left(\varepsilon-2 \mu_{1}\right) E_{2}(\varepsilon), \\
\left|u_{0}(\varepsilon)\right| & =\sqrt{\frac{1}{2}+\frac{\operatorname{sgn}\left(\varepsilon-2 \mu_{1}\right)\left(\frac{\varepsilon}{2}-\mu_{2}\right)}{2 E_{2}(\varepsilon)},} \\
\left|v_{0}(\varepsilon)\right| & =\sqrt{\frac{1}{2}-\frac{\operatorname{sgn}\left(\varepsilon-2 \mu_{1}\right)\left(\frac{\varepsilon}{2}-\mu_{2}\right)}{2 E_{2}(\varepsilon)},} \\
\Theta_{u}(\varepsilon) & =0, \quad \Theta_{v}(\varepsilon)=-\pi \theta\left(\varepsilon-2 \mu_{1}\right),
\end{aligned}
$$

which is consistent with pseudospin configuration [Eq. (B35)]. Since $\varepsilon_{+}=\varepsilon_{-}=2 \mu_{1}, E_{u}=E_{v}$ holds for any value of $\varepsilon$. It is obvious that Eq. (B27) will take the form of the phase II wave function after applying the transformation $\gamma \rightarrow \operatorname{sgn}(\varepsilon-$ $\left.2 \mu_{1}\right) \gamma$.
[1] T. Oka and H. Aoki, Phys. Rev. B 79, 081406(R) (2009).
[2] N. H. Lindner, G. Refael, and V. Galitski, Nat. Phys. 7, 490 (2011). 
[3] T. Kitagawa, T. Oka, A. Brataas, L. Fu, and E. Demler, Phys. Rev. B 84, 235108 (2011).

[4] Z. Gu, H. A. Fertig, D. P. Arovas, and A. Auerbach, Phys. Rev. Lett. 107, 216601 (2011).

[5] M. S. Rudner, N. H. Lindner, E. Berg, and M. Levin, Phys. Rev. X 3, 031005 (2013).

[6] A. Kundu, H. A. Fertig, and B. Seradjeh, Phys. Rev. Lett. 113, 236803 (2014).

[7] L. E. F. Foa Torres, P. M. Perez-Piskunow, C. A. Balseiro, and G. Usaj, Phys. Rev. Lett. 113, 266801 (2014).

[8] M. S. Foster, V. Gurarie, M. Dzero, and E. A. Yuzbashyan, Phys. Rev. Lett. 113, 076403 (2014).

[9] M. S. Foster, M. Dzero, V. Gurarie, and E. A. Yuzbashyan, Phys. Rev. B 88, 104511 (2013).

[10] Y. Dong, L. Dong, M. Gong, and H. Pu, Nat. Commun. 6, 6103 (2014).

[11] R. A. Barankov, L. S. Levitov, and B. Z. Spivak, Phys. Rev. Lett. 93, 160401 (2004).

[12] E. A. Yuzbashyan, O. Tsyplyatyev, and B. L. Altshuler, Phys. Rev. Lett. 96, 097005 (2006).

[13] R. A. Barankov and L. S. Levitov, Phys. Rev. Lett. 96, 230403 (2006).

[14] R. A. Barankov and L. S. Levitov, Phys. Rev. A 73, 033614 (2006).

[15] T. Iadecola, D. Campbell, C. Chamon, C.-Y. Hou, R. Jackiw, S.-Y. Pi, and S. V. Kusminskiy, Phys. Rev. Lett. 110, 176603 (2013).

[16] H. Dehghani, T. Oka, and A. Mitra, Phys. Rev. B 90, 195429 (2014); 91, 155422 (2015).

[17] L. D'Alessio and M. Rigol, Nat. Commun. 6, 8336 (2015).

[18] M. Dzero, E. A. Yuzbashyan, B. L. Altshuler, and P. Coleman, Phys. Rev. Lett. 99, 160402 (2007).

[19] J. R. Schrieffer, Theory of Superconductivity (Perseus Books, Reading, MA, 1983).

[20] Y. H. Wang, H. Steinberg, P. Jarillo-Herrero, and N. Gedik, Science 342, 453 (2013).

[21] R. Matsunaga, Y. I. Hamada, K. Makise, Y. Uzawa, H. Terai, Z. Wang, and R. Shimano, Phys. Rev. Lett. 111, 057002 (2013).

[22] R. Matsunaga, N. Tsuji, H. Fujita, A. Sugioka, K. Makise, Y. Uzawa, H. Terai, Z. Wang, H. Aoki, and R. Shimano, Science 345, 1145 (2014).
[23] There is a second important difference between tunneling and rf, besides the distribution function dependence of the latter. Because the rf probe is global, it conserves momentum. This leads to differences in terms of where gaps appear in the spectrum [24]; see Secs. III B and III C for more details.

[24] A. M. Essin and V. Gurarie, Phys. Rev. B 84, 125132 (2011).

[25] N. Tsuji, T. Oka, P. Werner, and H. Aoki, Phys. Rev. Lett. 106, 236401 (2011).

[26] E. A. Yuzbashyan and M. Dzero, Phys. Rev. Lett. 96, 230404 (2006).

[27] M. D. Caio, N. R. Cooper, and M. J. Bhaseen, arXiv:1504.01910 [cond-mat.str-el].

[28] E. A. Yuzbashyan, B. L. Altshuler, V. B. Kuznetsov, and V. Z. Enolskii, Phys. Rev. B 72, 220503(R) (2005).

[29] E. A. Yuzbashyan, B. L. Altshuler, V. B. Kuznetsov, and V. Z. Enolskii, J. Phys. A: Math. Gen. 38, 7831 (2005).

[30] E. Grosfeld, N. R. Cooper, A. Stern, and R. Ilan, Phys. Rev. B 76, 104516 (2007).

[31] This phase diagram was computed in [9]. The natural scale for $\Delta$ is set by the equilibrium transition $\Delta_{\mathrm{QCP}}$; the value of $\left(\Delta_{\mathrm{QCP}}\right)^{2}$ is determined by the fixed particle density $n$ and the condition $\mu=0$, up to a logarithmic dependence upon an ultraviolet cutoff $\Lambda$. To generate Fig. 1 , we set $\Lambda=50 \mathcal{E}_{F}=100 \pi n$. The same assumption is made throughout the paper to evaluate parameters of the dynamics (see Ref. [9] for details).

[32] Relative to the $s$-wave case, there is an additional complication for quenches in our 2D $p$-wave superfluid, if the initial state is a noninteracting Fermi liquid. In fact, for both $s$ - and $p$-wave cases, the Fermi step is a state of metastable equilibrium at the level of self-consistent mean field theory, and so does not evolve in time. In reality, quantum or thermal fluctuations will induce the instability of the normal state. For such a quench in the $p$-wave case, one would expect both $p+i p$ and $p-i p$ fluctuations; the subsequent evolution must track the interplay of both pairing channels [8]. We expect that the reduced BCS model retaining both channels is not integrable, so that the Lax technology used to solve the case of pure $p+i p$ order is not immediately applicable. This problem deserves further consideration.

[33] N. Read and D. Green, Phys. Rev. B 61, 10267 (2000).

[34] G. E. Volovik, The Universe in a Helium Droplet (Oxford University Press, Oxford, 2003). 